\title{
Absolute quantification of somatic DNA alterations in human cancer
}

\author{
Scott L Carter ${ }^{1,2}$, Kristian Cibulskis ${ }^{1,11}$, Elena Helman ${ }^{1,2,11}$, Aaron McKenna ${ }^{1,11}$, Hui Shen ${ }^{3,11}$, Travis Zack ${ }^{4,5,11}$, \\ Peter W Laird ${ }^{3}$, Robert C Onofrio ${ }^{1}$, Wendy Winckler ${ }^{1}$, Barbara A Weir ${ }^{1}$, Rameen Beroukhim ${ }^{1,5,6}$, \\ David Pellman ${ }^{7}$, Douglas A Levine ${ }^{8}$, Eric S Lander ${ }^{1,9,10}$, Matthew Meyerson ${ }^{1,5}$ \& Gad Getz ${ }^{1}$
}

\begin{abstract}
We describe a computational method that infers tumor purity and malignant cell ploidy directly from analysis of somatic DNA alterations. The method, named ABSOLUTE, can detect subclonal heterogeneity and somatic homozygosity, and it can calculate statistical sensitivity for detection of specific aberrations. We used ABSOLUTE to analyze exome sequencing data from 214 ovarian carcinoma tumor-normal pairs. This analysis identified both pervasive subclonal somatic pointmutations and a small subset of predominantly clonal and homozygous mutations, which were overrepresented in the tumor suppressor genes TP53 and NF1 and in a candidate tumor suppressor gene CDK12. We also used ABSOLUTE to infer absolute allelic copy-number profiles from 3,155 diverse cancer specimens, revealing that genome-doubling events are common in human cancer, likely occur in cells that are already aneuploid, and influence pathways of tumor progression (for example, with recessive inactivation of $N F 1$ being less common after genome doubling). ABSOLUTE will facilitate the design of clinical sequencing studies and studies of cancer genome evolution and intra-tumor heterogeneity.
\end{abstract}

Defining chromosome copy number and allele ratios is fundamental to understanding the structure and history of the cancer genome. Current genomic characterization techniques measure somatic alterations in a cancer sample in units of genomes (DNA mass).

\footnotetext{
${ }^{1}$ The Broad Institute of Harvard and MIT, Cambridge, Massachusetts, USA. ${ }^{2}$ Division of Health Sciences and Technology, MIT, Cambridge, Massachusetts, USA. ${ }^{3}$ USC Epigenome Center, Keck School of Medicine, University of Southern California, Los Angeles, California, USA. ${ }^{4}$ Biophysics Program, Harvard University, Cambridge, Massachusetts, USA. ${ }^{5}$ Divisions of Medical Oncology and Cancer Biology and Center for Cancer Genome Discovery, Dana-Farber Cancer Institute, Boston, Massachusetts, USA. ${ }^{6}$ Department of Medicine, Brigham and Women's Hospital, Boston, Massachusetts, USA. ${ }^{7}$ Howard Hughes Medical Institute, Department of Pediatric Oncology, Dana-Farber Cancer Institute, Children's Hospital, Department of Cell Biology, Harvard Medical School, Boston, Massachusetts, USA. ${ }^{8}$ Gynecology Service, Department of Surgery, Memorial Sloan-Kettering Cancer Center, New York, New York, USA. ${ }^{9}$ Department of Systems Biology, Harvard Medical School, Boston, Massachusetts, USA. ${ }^{10}$ MIT Department of Biology, Cambridge, Massachusetts, USA. ${ }^{11}$ These authors contributed equally to this work. Correspondence should be addressed to S.L.C. (scarter@broadinstitute.org) or G.G. (gadgetz@broadinstitute.org).
}

Received 25 October 2011; accepted 4 April 2012; published online 29 April 2012; doi: $10.1038 /$ nbt.2203
The meaning of such measurements is dependent on the tumor's purity and its overall ploidy; they are hence complicated to interpret and compare across samples. Ideally, copy number should be measured in copies per cancer cell. Such measurements are straightforward to interpret and, for alterations that are fixed in the cancer cell population, are simple integer values. This is considerably more challenging than measuring relative copy number in units of diploid DNA mass in a tumor-derived sample.

Measuring somatic copy-number alterations (SCNAs) on a relative basis is straightforward using microarrays ${ }^{1-5}$ or massively parallel sequencing technology $\mathrm{y}^{6,7}$; it has been the standard approach for copy-number analysis since the development of comparative genomic hybridization $(\mathrm{CGH})^{8}$.

Inferring absolute copy number is more difficult for three reasons: (i) cancer cells are nearly always intermixed with an unknown fraction of normal cells (tumor purity); (ii) the actual DNA content of the cancer cells (ploidy), resulting from gross numerical and structural chromosomal abnormalities, is unknown ${ }^{9-13}$; and (iii) the cancer cell population may be heterogeneous, perhaps owing to ongoing subclonal evolution ${ }^{14,15}$. In principle, one could infer absolute copy numbers by rescaling relative data on the basis of cytological measurements of DNA mass per cancer cell ${ }^{16-18}$, or by single-cell sequencing approaches ${ }^{15}$. However, such approaches are not suited to support initial large-scale efforts to comprehensively characterize the cancer genome ${ }^{19}$.

We began focusing on this issue several years ago, initially developing ad hoc techniques ${ }^{20,21}$. We subsequently developed the fully quantitative ABSOLUTE method and applied it to several cancer genome analysis projects, including The Cancer Genome Atlas (TCGA) project. ABSOLUTE provides a foundation for integrative genomic analysis of cancer genome alterations on an absolute (cellular) basis. We used these methods to correlate purity and ploidy estimates with expression subtypes and to develop statistical power calculations and use them to select well-powered samples for wholegenome sequencing in several published ${ }^{22-24}$, and numerous ongoing projects, including breast, prostate and skin cancer genome characterization. Recently, we extended ABSOLUTE to infer the multiplicity of somatic point-mutations in integer allelic units per cancer cell.

Our purpose here is to (i) present the mathematical inference framework of the ABSOLUTE method, as well as experimental validation of its predictions; (ii) apply it to analyze a large pan-cancer data set, enabling characterization of the incidence and timing of whole-genome doublings during tumor evolution; and (iii) describe 
a

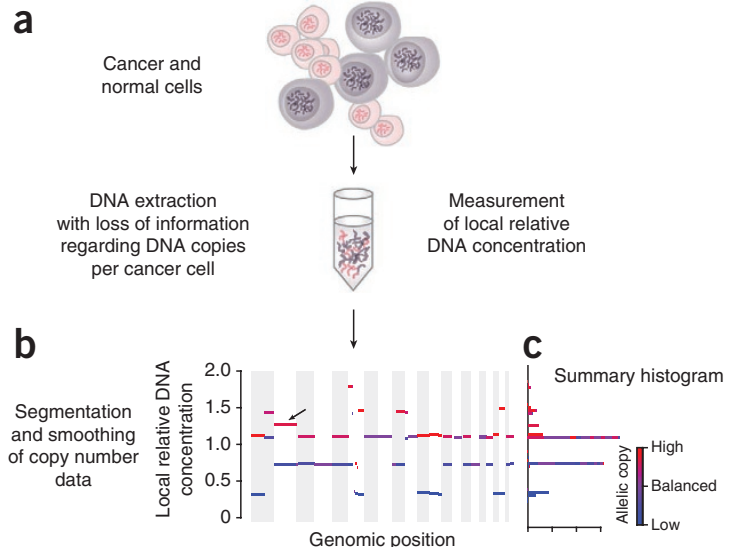

d

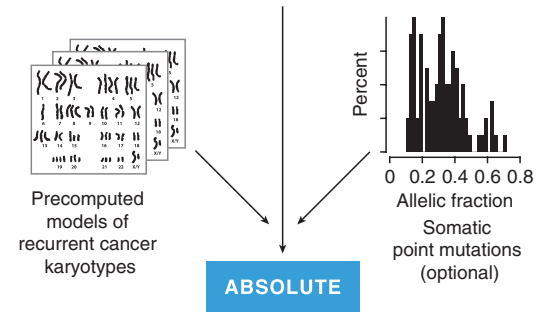

e

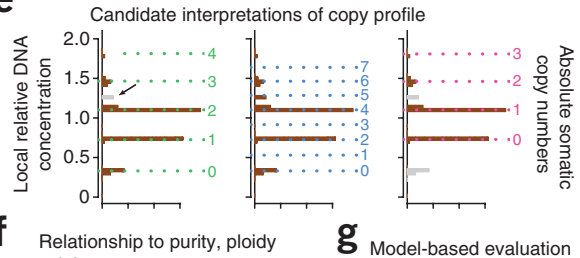

f Relationship to purity, ploidy
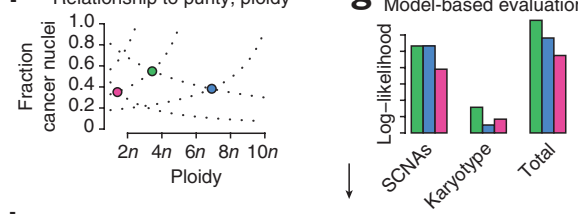

h

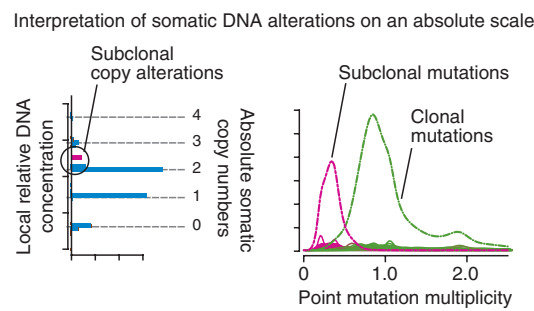

an integrated analysis of point-mutation and copy-number estimates and its application to ovarian carcinoma.

We describe three key mathematical features of ABSOLUTE. First, it jointly estimates tumor purity and ploidy directly from observed relative copy profiles (point mutations may also be used, if available). Second, because joint estimation may not be fully determined on a single sample, it uses a large and diverse sample collection to help resolve ambiguous cases. Third, it attempts to account for subclonal copy-number alterations and point mutations, which are expected in heterogeneous cancer samples.

We apply ABSOLUTE to conduct the first, to our knowledge, largescale 'pan-cancer' analysis of copy-number alterations on an absolute basis, across 3,155 cancer samples, representing 25 diseases with at least 20 samples each. The analysis reveals that whole-genome doubling events occur frequently during tumorigenesis, ultimately resulting in mature cancers descended from doubled cells bearing complex karyo-
Figure 1 Overview of tumor DNA analysis using ABSOLUTE. (a) A constant mass of DNA is extracted from a heterogeneous cell population consisting of cancer and normal cells. This DNA is profiled using either microarray or massively parallel sequencing technology, giving a genome-wide profile of DNA concentrations. (b) Genome-wide view of homologous copy ratios for a lung adenocarcinoma tumor sample processed using ABSOLUTE. The copy ratios for both homologous chromosomes are shown for each genomic segment with locally constant copy number. Color axis indicates distance between low (blue) and high (red) homologue concentration; segments where these are similar (allelic balance) are purple. (c) Homologous copyratio histogram. Copy ratios shown in $\mathbf{b}$ were binned at 0.04 resolution ( $y$ axis); the length of each block corresponds to the (haploid) genomic fraction ( $x$ axis) of each corresponding segment in $\mathbf{b}$. Several discrete SCNA peaks are visible, each corresponding either to an (unknown) integer copy state in the somatic clone or to a subclonal alteration. (d) To aid in the interpretation of potentially ambiguous data, ABSOLUTE uses precomputed statistical models of recurrence cancer karyotypes (left, Online Methods). Optionally, if somatic point mutation data are available (from sequencing of the DNA), then the allelic fractions (fraction of sequencing reads bearing the nonreference allele) of these mutations may be used help to interpret the DNA concentrations. (e) Three potential interpretations of the copy-ratio histogram (b) in terms of absolute copy numbers. Horizontal dotted lines indicate the copy ratios corresponding to the indicated absolute somatic copy-numbers. (f) Purity (fraction of tumor nuclei) and cancer-genome ploidy values corresponding to each interpretation in (e). Dotted lines denote potential solutions that share either $b$, the copy ratio associated with zero somatic copies (from upper left to lower right), or $\delta_{\tau}$, the spacing between consecutive integer copy levels (from lower left to upper right). Candidate solutions lie on the indicated grid of $b=2(1-\alpha) / D$ and $\delta_{\tau}=\alpha / D$ (equation (1)). (g) The log-likelihood (score) of each solution in terms of the SCNA fit of the observed copy ratios to integer absolute copy numbers and plausibility of the proposed karyotype. The highest-scoring solution (green) is identified by the combination of SCNA-fit and karyotype log-likelihood values. This interpretation implies subclonal gain of chromosome 2 (e, arrow). The SCNA score alone cannot distinguish between this and an additional solution (blue), in which the arrowed region is closer to an integer copy state, but the overall SCNA-fit score is equivalent to that of the first solution. (h) Interpretation of somatic DNA alterations on an absolute scale. Modeled SCNA copy states are shown (left). In addition, allelic fractions may be reinterpreted as average allelic copies per cancer cell (multiplicity), potentially revealing subclonal point mutations (right).

types. Despite evidence that genome doublings can result in genetic instability and accelerate oncogenesis ${ }^{13,25,26}$, the incidence and timing of such events had not been broadly characterized in human cancer.

We then describe how estimates of tumor purity and absolute copy number allow us to analyze allelic-fraction values (the fraction of non-reference sequencing reads supporting a mutation) to distinguish clonal and subclonal point mutations, and to detect macroscopic subclonal structure in an ovarian cancer sample. Clonal events may be classified as homozygous or heterozygous in the cancer cells, guiding interpretation of their function. In addition, the ability to quantify integer multiplicity of point mutations aids in the relative timing of segmental DNA copy-number gains, as multiplicity values of greater than one imply that the point mutation preceded copy gain of the locus. Controlling for tumor purity and local copy-number allow such timings to be calculated more generally than in the special case of copy-neutral loss of heterozygosity ${ }^{27}$. Finally, our data allow characterization of somatic cancer evolution with respect to wholegenome doubling, which we demonstrate in ovarian carcinoma and associate with clinicopathological values.

\section{RESULTS}

Inference of sample purity and ploidy in cancer-derived DNA A conceptual overview of ABSOLUTE is shown in Figure 1. When DNA is extracted from a mixed population of cancer and normal cells, the 
Figure 2 ABSOLUTE method validation and comparison. (a) FACS-based ploidy measurements versus inferred ploidy estimates for 37 primary tumor samples. Dashed line indicates $y=x$. RMSE: root mean squared error. $P$-values were calculated on the squared errors using the paired one-sided Wilcoxon test $\left({ }^{*}, P<0.05\right.$; $* *, P<0.001$ ). (b) SKY-based ploidy measurements versus inferred ploidy estimates for 33 cancer cell lines. Data are displayed as in a. (c) Estimated purity of the 33 cell lines shown in $\mathbf{b}$. Dashed horizontal line indicates the true purity (1.0). (d) Cancer-normal DNA mixing experiment results for two cell lines. DNA from each cancer cell line was mixed with DNA from the matched B-lymphocyte in varying proportions ( $x$ axis). Top, predicted versus true DNA mixing fractions compared to the $y=x$ line (dashed). Bottom, predicted cancer cell line ploidy versus mixture purity. The copy profile of several samples was misinterpreted (x's); these points were not included in the RMSE calculations. Ploidy estimates were generally consistent with previous SKY analysis of these cell lines: http://www.path.cam.ac.uk/ pawefish/ cell\%20line\%20catalogues/breast-cell-lines.htm (e) Leukocyte methylation signature enrichment in tumors of histologically underestimated purity. HGS-OvCa samples are shown grouped according to the indicated histological purity estimates $(x \text { axis })^{34}$. Black horizontal lines indicate the median purity of each group, as estimated by ABSOLUTE. The color of each point corresponds to the degree to which that sample's methylation profile resembled that of purified leukocytes.
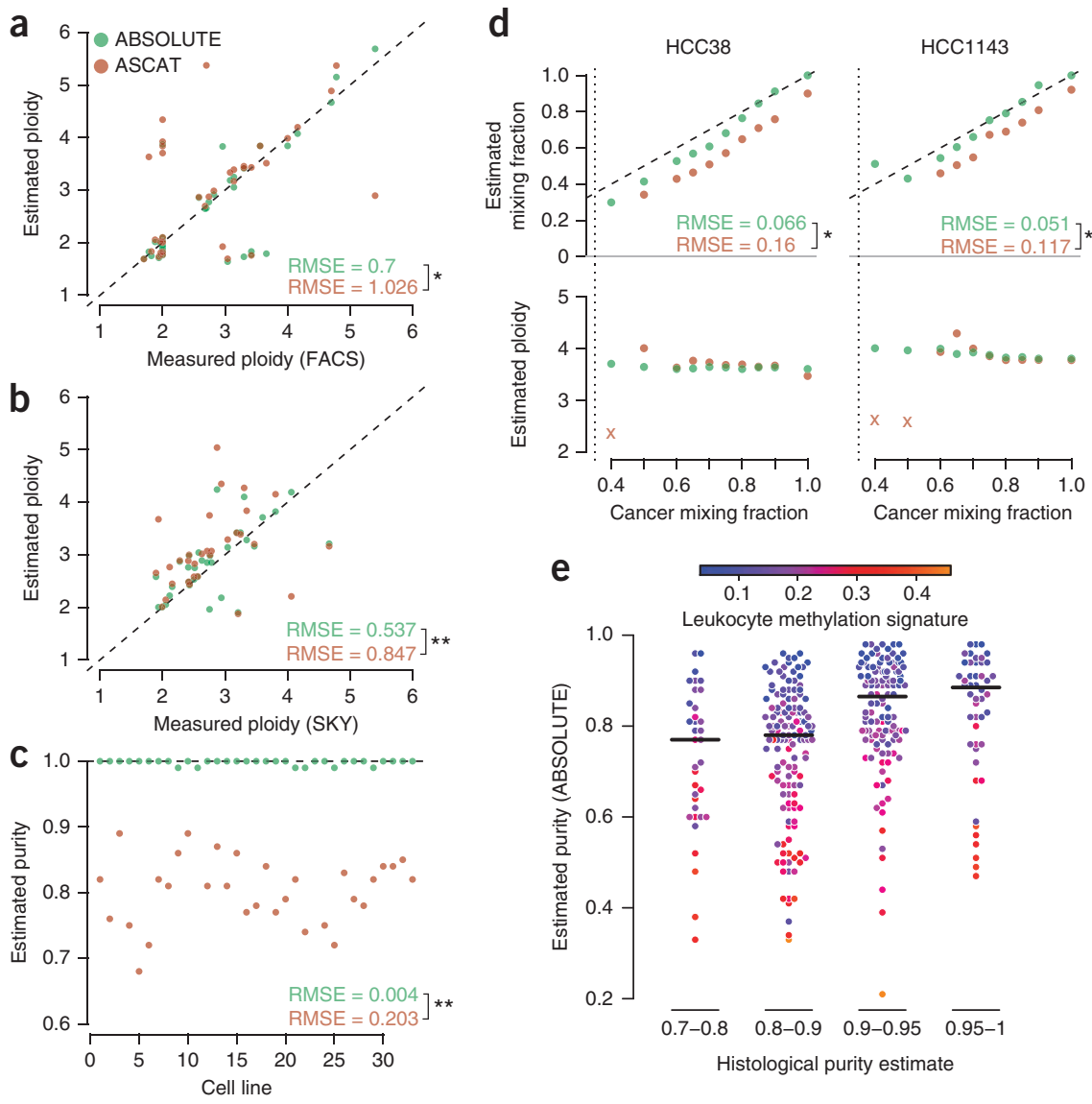

information on absolute copy number per cancer cell is lost. The purpose of ABSOLUTE is to infer this information from the population of mixed DNA. This process begins with the generation of segmented copy-number data, which is input to the ABSOLUTE algorithm together with precomputed models of recurrent cancer karyotypes and, optionally, allelic fraction values for somatic point mutations. The output of ABSOLUTE then provides inferred information on the absolute cellular copy number of local DNA segments and, for point mutations, the number of mutated alleles (Fig. 1).

We begin by describing the inference framework used in ABSOLUTE. Suppose a cancer-tissue sample consists of a mixture of a proportion $\alpha$ of cancer cells (assumed to be monogenomic-that is, with homogenous SCNAs in the cancer cells) and a proportion $(1-\alpha)$ of contaminating normal (diploid) cells. For each locus $x$ in the genome, let $q(x)$ denote the integer copy number of the locus in the cancer cells. Let $\tau$ denote the mean ploidy of the cancer-cell fraction, defined as the average value of $q(x)$ across the genome. In the mixed cancer sample, the average absolute copy number of locus $x$ is $\alpha \mathrm{q}(x)+$ $2(1-\alpha)$ and the average ploidy $(D)$ is $\alpha \tau+2(1-\alpha)$, measured in units of haploid genomes.

The relative copy number $(R)$ of locus $x$ is therefore:

$$
R(x)=(\alpha \mathrm{q}(x)+2(1-\alpha)) / D=(\alpha / D) \mathrm{q}(x)+(2(1-\alpha) / D)
$$

Because $\mathrm{q}(x)$ takes integer values, $R(x)$ takes discrete values. The smallest possible value is $(2(1-\alpha) / D)$, which occurs at homozygously deleted loci and corresponds to the fraction of DNA from normal cells. The spacing between values $(\alpha / D)$ corresponds to the concentration ratio of alleles present at one copy per cancer cell and zero copies per normal cell. Notably, if a cancer sample is not strictly clonal, copy-number alterations occurring in substantial subclonal fractions will appear as outliers from this pattern (Fig. 1b,e, arrows). Similar considerations have formed the basis for algorithms to infer purity and ploidy using allelic copy-ratios derived from singlenucleotide polymorphisms (SNP) microarrays ${ }^{28-33}$.

We extend absolute copy inference to encompass somatic point mutations as follows:

$$
F(x)=\left(\alpha \mathrm{s}_{\mathrm{q}}(x)\right) / D_{\mathrm{s}}=\left(\alpha / D_{\mathrm{s}}\right) \mathrm{s}_{\mathrm{q}}(x)
$$

Here, $\mathrm{s}_{\mathrm{q}}$ represents the multiplicity of the point mutation, in integer values per cancer cell (which cannot exceed $\mathrm{q}(x)$ ), and $D_{\mathrm{s}}=\alpha q(x)+$ $2(1-\alpha)$. The values of $F(x)$ correspond to the expected fraction of sequencing reads that support the mutation, which depend on the sample purity and absolute somatic copy number at the mutant locus, $\mathrm{q}(x)$.

The ABSOLUTE algorithm examines possible mappings from relative to integer copy numbers by jointly optimizing the two parameters $\alpha$ and $\tau$ (Fig. 1e-g; Supplementary Fig. 1; Online Methods equation (5)). In many cases, several such mappings are possible, corresponding to multiple optima.

To help resolve ambiguous cases, we used recurrent cancerkaryotype models based on large data sets (Supplementary Fig. 2; Online Methods equation (8) to identify the simplest (that is, most common) karyotype that can adequately explain the data. This method favors simpler solutions, while preserving the flexibility to identify unexpected karyotypes given sufficient evidence from the copy profile. Indeed, several unusual karyotypes, including near-haploid $(<1.2 n)$ and hyperaneuploid $(>6 n)$ genomes, were identified using ABSOLUTE (Supplementary Fig. 3). 
a
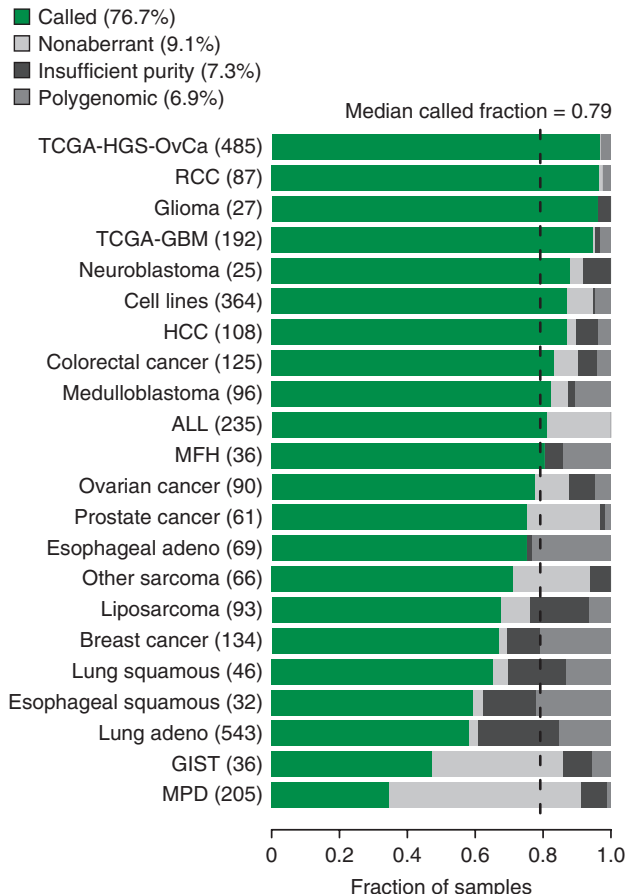

Figure 3 Pan-cancer application of ABSOLUTE. (a) ABSOLUTE result types: (i) called, unique purity/ploidy solution; (ii) nonaberrant, sample has no detectable somatic copynumber alterations; (iii) insufficient purity, insufficient fraction of cancer cells; (iv) polygenomic, discrete copy-ratio levels could not be determined. (b) Distribution of estimated tumor purity for several data sets. We note that, because heavily

contaminated tumors are difficult to call using ABSOLUTE, several of these distributions are biased toward higher purity samples. (c) Distribution of estimated cancer genome ploidy for several datasets. Because tumors without SCNAs cannot be called using ABSOLUTE, these distributions do not incorporate the prevalence of such samples. (b,c) The number of called tumor samples is each group is shown in parentheses. b

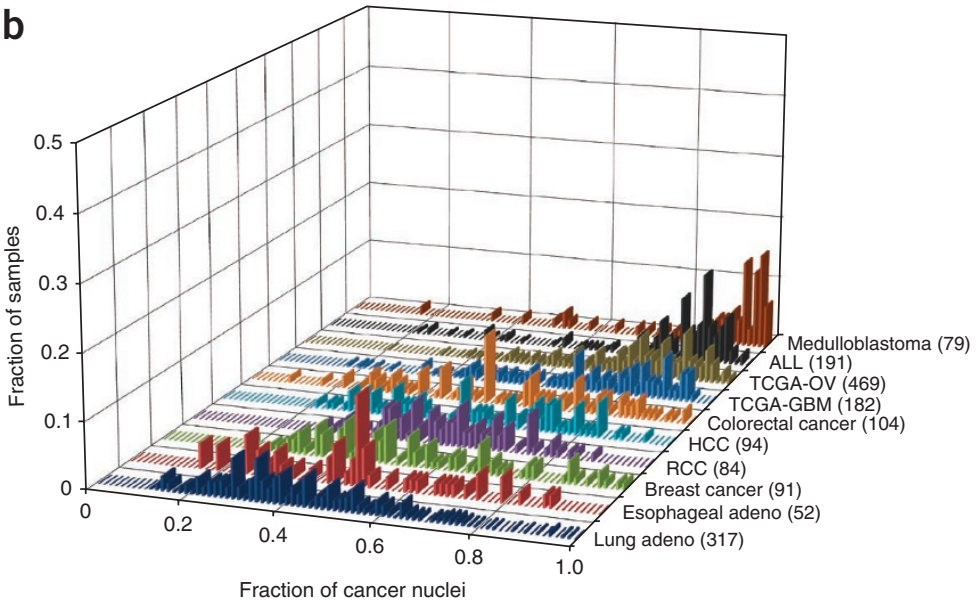

C

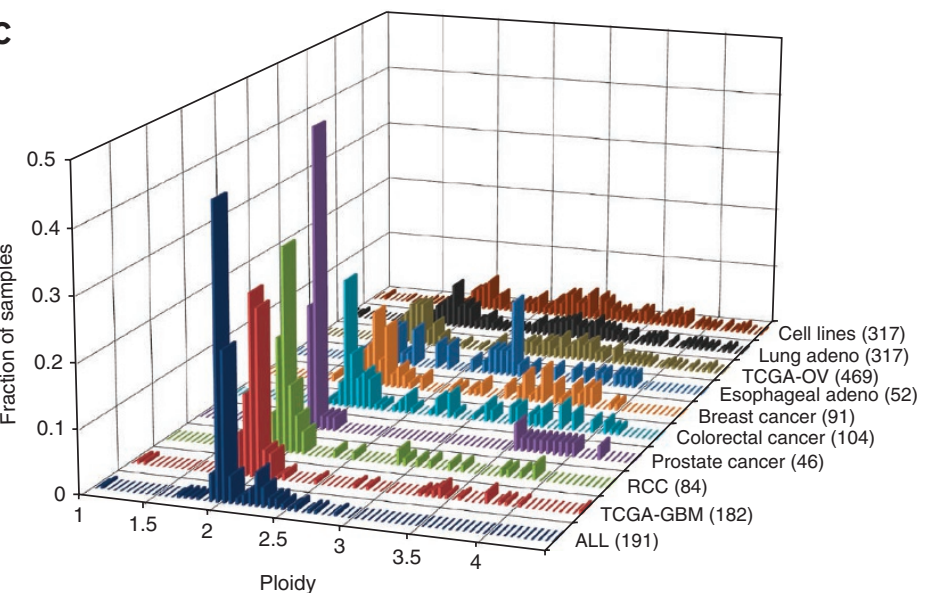

Our implementation supports copy-number inference from either total or allelic copy-ratio data, such that array-CGH, SNP microarray or massively parallel sequencing data may be used. ABSOLUTE is available for download at http://www.broadinstitute.org/cancer/cga/ABSOLUTE.

\section{Validation}

We validated the purity and ploidy predictions made by ABSOLUTE on Affymetrix SNP microarray data using several approaches: (i) direct ploidy measurement of 37 TCGA ovarian carcinoma samples by fluorescence-activated cell sorting ${ }^{34}$ (Fig. 2a); (ii) measurement of ploidy for 33 NCI60 cell lines based on spectral karyotyping ${ }^{35}$ (Fig. 2b,c); and (iii) DNA-mixing experiments, in which cancer cell lines were mixed with paired normal B lymphocyte-derived DNAs in varying mass proportions (Fig. 2d, Online Methods). We also evaluated a related computational method, ASCAT ${ }^{31}$, on these data (Fig. $\mathbf{2 a - d}$ and Supplementary Note). Although the results were broadly concordant with our estimates, ABSOLUTE achieved significantly more accurate results (Fig. 2a-d) on our validation data. Notably, we observed an apparent bias by ASCAT to underestimate the cancer cell fraction (Fig. 2 c,d), consistent with previous reports applying ASCAT in similar mixing experiments using Illumina SNP arrays ${ }^{31}$ (Fig. S4 therein).

Notably, the purity estimates produced by ABSOLUTE appeared to be more accurate for the bulk tumor than those derived from histological examination of frozen tumor sections (Online Methods, Fig. 2e). Estimates of the proportion of contaminating normal cells for 458 ovarian carcinoma samples ${ }^{34}$ produced by ABSOLUTE were strongly correlated with a molecular signature of genomic methylation (Online Methods) seen in leukocytes $\left(r^{2}=0.59, P<2.2 \times 10^{-16}\right.$, Fig. 2e), but only weakly correlated with estimates of contamination from histological examination $\left(r^{2}=0.1, P=2.4 \times 10^{-12}\right.$; Online Methods; Fig. $2 \mathrm{e} x$-axis scale, Supplementary Fig. 4).

\section{Estimation of tumor purity and ploidy across cancer types}

We used ABSOLUTE to analyze allelic copy-ratio profiles derived from SNP arrays from 3,155 cancer samples, comprising 2,791 tissue specimens and 364 cancer cell lines. This yielded predicted purity and ploidy values (Supplementary Table 1) and the segmented absolute allelic copy number of each tumor (Supplementary Table 2). The samples came from two TCGA pilot studies describing glioblastoma multiforme (GBM; 192 samples) ${ }^{21}$ and ovarian carcinoma (488 samples) ${ }^{34}$, as well as 2,445 profiles incorporated from a previous pan-cancer copy-number analysis ${ }^{36}$ (Online Methods; see Supplementary Table 1 for characteristics of each tumor sample). A minority of these samples (519 or 16.4\%) could not be analyzed because they lacked clearly identifiable SCNAs, either because they were nearly euploid (nonaberrant), or were excessively contaminated with normal cells (insufficient purity) (Fig. 3a). Although sequencing data for somatic point mutations may have resolved these cases, such data were not available for the majority of samples in this cohor ${ }^{36}$. 
Figure 4 Characterization of subclonal evolution in ovarian cancer by integrative analysis of SNP array and whole-exome sequencing data. (a) Histogram of allelic fraction (alternate/ total read-count) values for 29,628 somatic point-mutations detected in 214 primary HGSOvCa samples ${ }^{34}$. (b) Allelic fractions for the mutations shown in a were converted to point estimates of average allele-counts per cancer cell (cellular multiplicity; $x$ axis) by correcting for sample purity and local copy numbers. Subclonal mutations were identified using the model defined in equation (12). (c) The fraction of each of the six distinguishable nucleotide substitutions for clonal versus subclonal pointmutations. The solid gray line indicates $y=x$. RMSE, root mean squared error. (d) Tumor SCNA profile with modeled absolute copy numbers. Regions of normal homologous copy number $=1$ are grayed out, clonal SCNAs are brown.

Subclonal SCNAs (light blue) appear in several clusters. Colored arrows indicate subclonal

SCNAs present at equivalent cell fraction. (e) Point mutation allelic-fraction profile. Each solid curve corresponds to a single mutation, with the density according to the posterior (Beta) distribution implied by the observed allelic fraction and local read depth. Color indicates degree of classification as clonal or subclonal, as in $\mathbf{b}$. Dashed curves indicate summed density of individual posteriors. (f) SCNAs from $\mathbf{d}$ and point mutations from e were rescaled to units of cancer cell fraction. Subclonal cancer cell fractions of $\sim 0.2,0.3$ and 0.6 are supported both by SCNAs and point mutations (purple, blue and orange arrows, respectively; see corresponding copy ratios in $\mathbf{d}$ ). Analysis of distinct subclonal populations in $\mathbf{d}-\mathbf{f}$ was performed on HGS-OvCa sample TCGA-24-1603 (purity = 0.96, ploidy = 1.75).

For the 2,636 samples with detectable SCNAs, ABSOLUTE provided purity and ploidy calls for $92 \%$ of cases, and designated the remaining samples as 'polygenomic' (genomically heterogeneous) (Fig. 3a), (Online Methods and Supplementary Fig. 5). The fraction of called samples varied by disease type, from $34.6 \%$ (myeloproliferative disease; mostly nonaberrant genomes) to $96.7 \%$ (ovarian carcinoma; $100 \%$ aberrant genomes), with a median call-rate of $79.2 \%$ (Fig. 3a).

The distributions of estimated purity varied among cancer types, with the tested lung, esophageal and breast cancer samples being the least pure on average in our data set (Fig. 3b). The effect of contamination was readily visible in the copy ratios of impure tumor types (Supplementary Fig. 6). Distributions of estimated ploidy (Fig. 3c) were qualitatively consistent with those derived from previously obtained cytological data for each tumor type ${ }^{13}$.

\section{Power for detection of somatic point-mutations by sequencing}

Both tumor purity and ploidy affect the local depth of sequencing necessary to detect point mutations. For example, suppose that a region is present at six copies with only one copy carrying a mutation in a sample that has $50 \%$ contamination with normal cells. In this case, only one of eight alleles at this locus (six from the cancer cells and two from the normal cells) carry the mutation (Supplementary Fig. 7a). We therefore expect that the mutation will be observed in only $12.5 \%$ of reads. Given this allelic fraction, local sequence coverage of 33 -fold is required to detect the mutation with $80 \%$ sensitivity, assuming a sequencing error rate of $10^{-3}$ per base and a falsepositive rate controlled at $<5 \times 10^{-7}$ (Online Methods, equation (9); Supplementary Fig. 7b).

Using ABSOLUTE's estimates of purity and genome-wide integer copy numbers, we can calculate the required coverage for powered detection of mutations present at specified allelic multiplicity per cancer cell. Similar considerations apply to detecting subclonal mutations, present in a fraction of cancer cells, by using fractional multiplicities (Supplementary Fig. 7c). We note that consideration of tumor purity in units of cells, rather than DNA fraction, is preferred for devising power calculations for sequencing experiments, because many somatic alterations of interest are expected to occur at a single copy per cancer cell.

We analyzed the distribution of purity and ploidy values in cancer samples analyzed for allelic copy number ${ }^{21,34,36}$ to determine an appropriate depth of sequencing coverage needed to detect clonal mutations with power 0.8 in each sample. For this purpose, we calculated the number of reads needed to detect a mutation present in one copy, at a locus present at the average copy number, given the sample's purity. (One could alternatively choose a particular percentile on the copy-number distribution.) For such a locus, we found that $30 \times$ local coverage would suffice for most samples (Supplementary Fig. 7d). By contrast, a locus of average copy number with a mutation carried in a subclone at 0.2 cancercell fraction would require coverage of $\sim 100$-fold to allow detection in about half of the samples (Supplementary Fig. 7e). Using these calculations and the distribution of local coverage along the genome (which depends on the specific sequencing technology), one can determine the fraction of the genome (e.g., $>80 \%$ power in $>80 \%$ of the genome).

We then examined whole-exome sequencing data $(\sim 150 \times$ average coverage) from 214 TCGA ovarian carcinoma samples ${ }^{34}$ to determine whether detection power was related to the number of mutations loci for which the local coverage provided at least $80 \%$ power to detect mutations present at single copy in a subclone present at 0.05 cancercell fraction. Those samples with the lowest proportion of such wellpowered loci tended to be those in which the fewest such mutations were detected $\left(r^{2}=0.24, P=2.7 \times 10^{-13}\right.$; Supplementary Fig. 7 f $)$, suggesting that the failure to find such mutations was due to the lack of power. This result also demonstrates the importance of power calculations for characterization of the subclonal frequency spectrum.

\section{Multiplicity analysis of somatic point-mutations}

We next used ABSOLUTE to convert the allelic fraction of mutations to cellular multiplicity estimates. For this purpose, we examined average coverage necessary to obtain sufficient power in a predefined actually observed. For each sample, we calculated the proportion of 
a

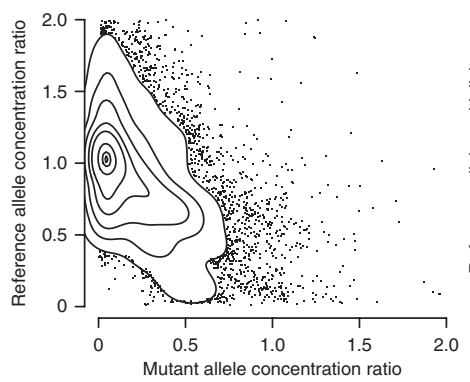

b

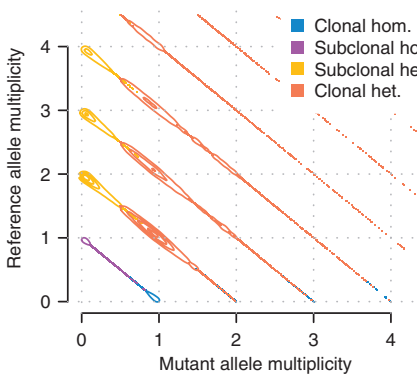

C

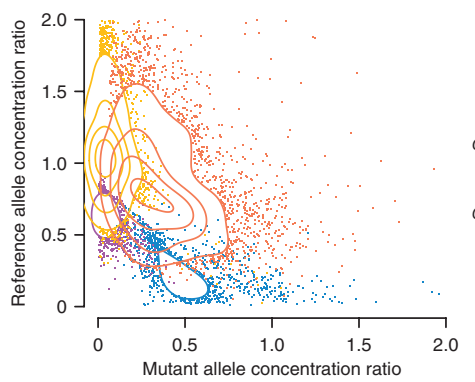

d

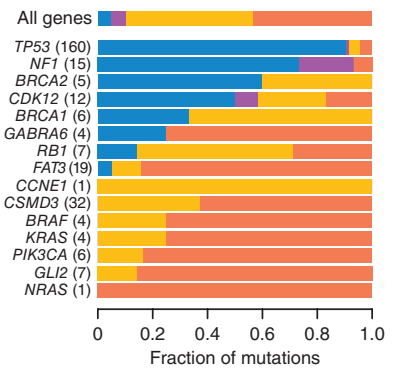

Figure 5 Classification of somatic mutations by multiplicity analysis in 214 primary HGS-OvCa tumor samples. (a) Empirical density estimate of allelic concentration ratios, which are obtained by multiplication of the allelic fraction by the copy ratio at that locus. (b) Density estimate of allelic multiplicity estimates, as in Figure 4b, for reference versus mutant allele. Mutations were classified into the four indicated categories according to their mutant and reference allele multiplicity. (c) The density estimates of allelic concentration ratios for each of the four mutation classes in $\mathbf{b}$ are shown superimposed. (d) Mutation classification

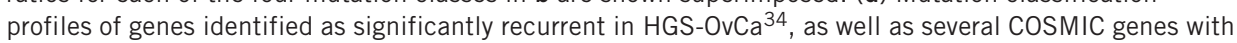
previously observed mutations in these data. Note that only individual point mutations were considered here; the possibility of recessive inactivation through multiple mutations (compound heterozygosity) was not considered. (e) Histograms of gene classification fractions for 1,412 genes having at least five recurrent mutations. Dotted vertical lines denote the 5th (top) and 95th (other) percentiles of each distribution. No mutations occurring at multiplicity $>1$ were observed in NF1 (data not shown).

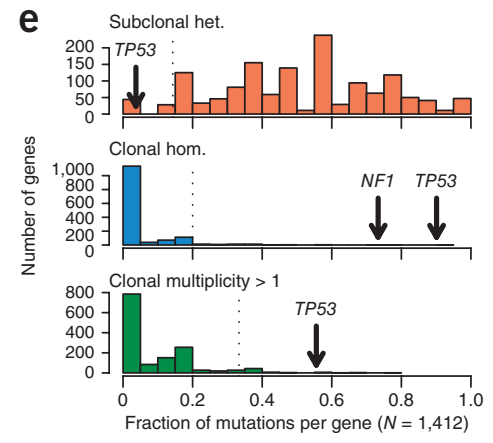

29,268 somatic mutations identified in whole-exome hybrid capture Illumina sequencing ${ }^{37}$ data from 214 ovarian carcinoma tumornormal pairs ${ }^{34}$ (Fig. 4a). Tumor purity, ploidy and absolute copynumber values were obtained from Affymetrix SNP6.0 hybridization data on the same DNA aliquot that was sequenced, allowing the rescaling of allelic fractions to units of multiplicity (Fig. 4a,b; Online Methods, equation (12)).

This procedure identified pervasive subclonal point-mutations in ovarian carcinoma samples. Although many of the mutations were clustered around integer multiplicities, a substantial fraction occurred at multiplicities substantially less than one copy per average cancer cell, consistent with subclonal multiplicity (Fig. 4b)).

Several lines of evidence support the validity of these subclonal mutations, including Illumina resequencing of an independent wholegenome amplification aliquot, which confirmed both their presence (Supplementary Fig. 8a,b), and that their allelic fractions corresponded to subclonal multiplicity values (Supplementary Fig. 8c,d). In addition, the mutation spectrum seen for clonal and subclonal mutations was similar (root mean squared error (RMSE) = 0.04, Fig. 4c), consistent with a common mechanism of origin. Power calculations showed that these samples were at least $80 \%$ powered for detection of subclonal mutations occurring in cancer-cell fractions ranging from 0.1 to 0.53 , with a median of 0.19 (Supplementary Fig. 7e).

The distribution of subclonal multiplicity was similar in the majority of samples (Fig. 4b); it rapidly increased at the sample-specific detection limit and then decreased in a manner approximated by an exponential decay in the multiplicity range of 0.05 to 0.5 when pooling across all samples. In contrast, the high-grade serous ovarian carcinoma (HGS-OvCa) sample TCGA-24-1603 (Fig. 4d-f) showed evidence for discrete 'macroscopic subclones'. Rescaling of subclonal SCNAs (Fig. 4d) and point mutations (Fig. 4e) to units of cancer cell fraction (Fig. 4f) revealed discrete clusters near fractions $0.2,0.3$ and 0.6 (Fig. 4f), implying the alterations within each cluster likely co-occurred in the same cancer cells. We note that this combination of cell fractions sums to more than one, implying that at least one of the detected subclones was nested inside another.
We next used ABSOLUTE to analyze the multiplicity of both the reference and alternate alleles in order to classify point mutations as either heterozygous or homozygous in the affected cell fraction (Fig. 5a-c). We considered 15 genes with mutations recently identified in these data ${ }^{34}$, including five known tumor suppressor genes and five oncogenes (Fig. 5d). The frequency of homozygous mutations in known tumor suppressor genes and oncogenes was significantly different, with a significantly elevated fraction of homozygous mutations in the tumor suppressor genes $(P=0.006$, Fig. 5d) and no homozygous mutations in the oncogenes $(P=0.012$, Fig. $5 d)$. This result provides evidence supporting $C D K 12$ as a candidate tumor suppressor gene in ovarian carcinoma ${ }^{34}$, since 7 of 12 CDK12 mutations were homozygous $\left(P=6.5 \times 10^{-5}\right.$; Fig. 5d).

Overall, TP53 had among the greatest fraction of clonal, homozygous and 'multiplicity $>1$ ' mutations of any gene in the coding exome (Fig. 5e), demonstrating the clear identification of a key initiating event in HGS-OvCa carcinogenesis ${ }^{38}$ directly from multiplicity analysis.

\section{Whole-genome doubling occurs frequently in human cancer}

For many cancer types, the distribution of total copy number (ploidy) was markedly bi-modal (Fig. 3c), consistent with chromosome-count profiles derived from SKY ${ }^{10,13}$. Although these results are consistent with whole-genome doubling during their somatic evolution, it has been difficult to rule out the alternative hypothesis that evolution of high-ploidy karyotypes results from a process of successive partial amplifications ${ }^{12}$.

To study genome doublings, we used homologous copy-number information - that is, the copy numbers, $b_{i}$ and $c_{i}$, of the two homologous chromosome segments at each locus. By looking at the distributions of $b_{i}$ and $c_{i}$ across the genome, we could draw inferences regarding genome doubling. Immediately following genome doubling, both $b_{i}$ and $c_{i}$ would be even numbers. Following the loss of a single copy of a region, the larger of $b_{i}$ and $c_{i}$ will remain even, but the smaller would become odd. In fact, when we looked at highploidy samples, we discerned that the higher of $b_{i}$ and $c_{i}$ was usually even throughout the genome, consistent with their having arisen by 
Figure 6 Incidence and timing of whole-genome doubling events in primary cancers. $(\mathbf{a}, \mathbf{b})$ Ploidy estimates were obtained from ABSOLUTE. Mean homolog imbalance was calculated as the average difference in the homologous copy numbers at every position in the genome. Genome doubling status was inferred from the homologous copy numbers. (c) Frequency of genome doubling by cancer type. MPD, myeloproliferative disease;

$A L L$, acute lymphoblastic leukemia; GBM, glioblastoma multiforme; RCC, renal cell carcinoma; HCC, hepatocellular carcinoma; HGS-OvCa, high-grade serous ovarian carcinoma. (d) LOH (loss of heterozygosity) was defined as 0 homologous copies. Amplification was defined as $>1$ homologous copy for samples with 0 genome doublings, and as $>2$ homologous copies for those with 1 genome doubling. Calls were made based on the modal allelic copy numbers of each chromosome arm. Dashed lines indicate $y=x$. (e) SCNAs, defined as regions differing from the modal absolute copy number of each sample, were binned at adaptive resolution to maintain 200 SCNAs per bin, and renormalized by bin length. The value in each bin was further divided by the number of tumor samples in each genome doubling class, indicated by color as in $\mathrm{a}$. The black line indicates slope $=-1$. Linear regression models were fit independently for each class using SCNAs $0.5<x<20 \mathrm{Mb}$. This resulted in fitted slope values of $-1.05,-0.96$ and -0.88 for 0,1 and $>1$ genome doublings, respectively (data not shown). a

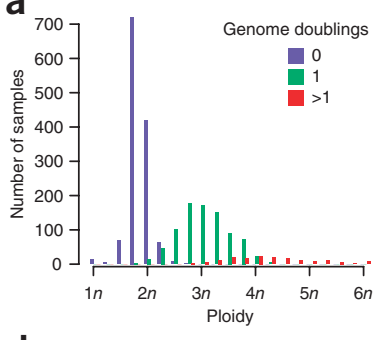

b

d Esophageal adenocarcinoma HGS-OvCa GBM

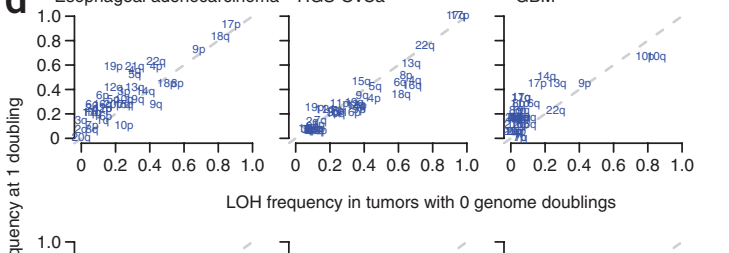

产 1.0

0.6
0.4
0.2
0

$\begin{array}{llllllllllllllllllllll}0.2 & 0.4 & 0.6 & 0.8 & 1.0 & 0 & 0.2 & 0.4 & 0.6 & 0.8 & 1.0 & 0 & 0.2 & 0.4 & 0.6 & 0.8 & 1.0\end{array}$

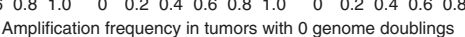

C
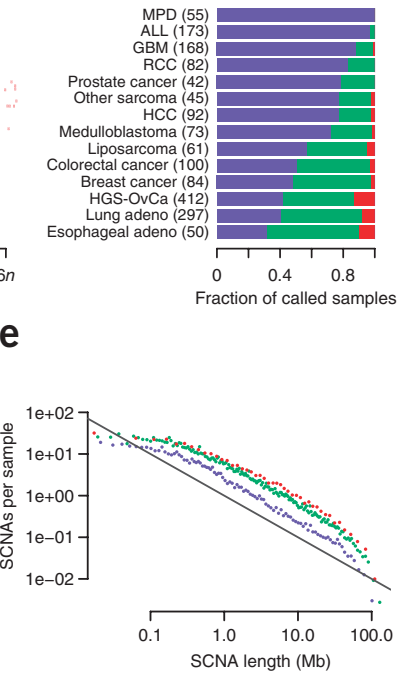

doubling of the entire genome (Supplementary Fig. 9). Using simulations, we found that the observed profiles were unlikely to arise owing to SCNAs occurring in serial fashion at multiple independent chromosomes $\left(P<10^{-3}\right)$.

Using such information, we classified samples into three groups, which we interpreted as corresponding to 0,1 and $>1$ genome doubling events in the clonal evolution of the cancer. These three groups had modal ploidy values of 1.75, 2.75 and 4.0, respectively (Fig. 6a), and also segregated into three clusters by ploidy and mean homologous copy-number imbalance (Fig. 6b). We interpreted this as evidence of SNCAs occurring with net losses, interspersed with the genome doublings. This process resulted in intermediate ploidy values for the doubled clones $(2.2-3.4 n)$, with pervasive imbalance of homologous chromosomes (Fig. 6b).

The frequency of genome doubling varied across tumor types (Fig. 6c), reflecting differences in disease-specific biology and clinical progression status. Hematopoietic neoplasms (myeloproliferative disease, acute lymphoblastic leukemia) had nearly no doubling events, whereas glioblastoma multiforme, renal cell carcinoma, prostate cancer, various sarcomas, hepatocellular carcinoma and medulloblastoma all had $25 \%$ incidence of doubling. Genome doubling was more common in epithelial cancers, with colorectal, breast, lung, ovarian and esophageal cancers all having $>50 \%$ incidence of doubling (Fig. 6c). Esophageal adenocarcinoma had the greatest doubling incidence, consistent with previous reports of frequent $4 n$ populations at various stages of Barrett's esophagus progression ${ }^{39,40}$.

\section{Specific aneuploidies precede genome doubling}

We then used ABSOLUTE to infer the temporal order of genome doubling in tumorigenesis, relative to SNCAs involving specific chromosome arms. In many cancer types, the fixation of arm-level SCNAs was inferred to occur before genome doubling, because both doubled and nondoubled samples had similar frequencies of specific arm-level SNCAs (Fig. 6d and Supplementary Fig. 10).
In glioblastoma multiforme samples, loss of heterozygosity involving chromosomes 9 and 10, and gains of chromosome 7 occurred at equivalent frequencies (Fig. 6d), demonstrating that the most common broad SCNAs in glioblastoma multiforme occur before genome doubling. Gain of chromosomes 19 and 20 was nearly exclusive to nondoubled samples, and several arms had greater frequency of loss of heterozygosity in doubled samples (Fig. 6d), suggesting that additional biological differences underlie these samples.

Because ABSOLUTE could not distinguish between ploidy $2 \mathrm{~N}$ and $4 \mathrm{~N}$ in cases with no observed SCNAs, we discarded such nonaberrant samples from our analysis (Fig. 3a). For many tumor types, such cases were rare, due to the tendency for chromosomal losses after doubling (Figs. 3c and 6a,b and Supplementary Fig. 9). The representation of specific cancer subtypes may be biased by differences in ascertainment, however.

In contrast to broad chromosomal alterations, focal SCNA events occurred at greater frequency in doubled genomes (Fig. 6e). Consistent with previous reports ${ }^{36,41,42}$, the observed frequency of focal SCNAs as a function of their length $(L)$ followed power-law scaling: $P(L) \propto L^{-\alpha}$, for $L>0.5 \mathrm{Mb}$ (Fig. 6e). Genome doubling was associated with a larger overall number of SCNAs; however, we obtained estimates of $\alpha$ near 1 for each group (Fig. 6e), suggesting that the mechanism(s) by which they were generated did not greatly depend on ploidy.

Genome doubling influences progression of ovarian carcinoma We next sought to correlate whole-genome doubling occurrence in high-grade serous ovarian carcinoma with other genetic and clinical features. Genome-doubled samples showed a higher incidence of heterozygous mutations, but correcting for sample ploidy removed this effect (Fig. 7a), suggesting that the per-base mutation rates are equivalent. Clonal mutations at multiplicity $>1$ were approximately tenfold more prevalent in doubled samples; many of these events likely occurred before the doubling event. Genome-doubled samples had significantly lower frequencies of both of homozygous deletions (Fig. 7b) and of clonal homozygous mutations (Fig. 7c). We expect 


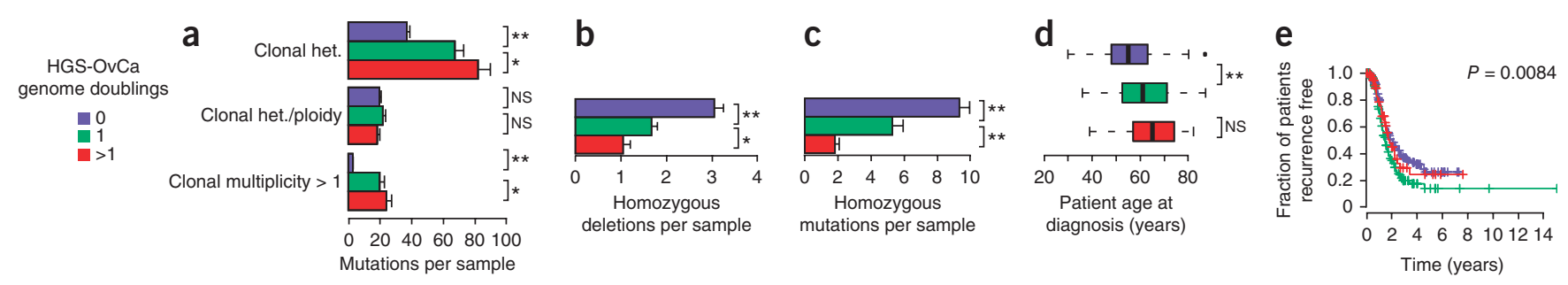

Figure 7 Genetic and clinical associations with genome doubling in primary HGS-OvCa samples. (a-c) Number of mutations in indicated classes as a function of genome doublings. Error bars indicate s.e.m. (a-d) $P$-values were calculated with the two-sided Wilcoxin rank-sum test. (e) $P$-values were calculated using the log-rank test. Colors correspond to putative genome-doubling status, as indicated. ${ }^{*}, P<10^{-5} ;{ }^{*}, P<0.05 ;$ NS, $P>0.05$.

that many of the observed homozygous alterations in the doubled samples were fixed before genome doubling.

The lower incidence of homozygous mutations in genome-doubled samples may reflect the fact that more events are required to render a mutation homozygous in a genome-doubled sample (although the effect may be partially offset by a possible increase in genetic instability following doubling, for example, by centrosome duplication ${ }^{43}$ ). These considerations suggest that genome-doubled samples evolve by means of distinct trajectories, because inactivation of tumor suppressors may occur less frequently after doubling.

We note that 13 of the 15 detected point mutations in the tumor suppressor NF1 occurred in the 93 ovarian samples that had not undergone genome doubling $(P=0.002$; Fisher's exact test), and these mutations were uniformly homozygous (data not shown). This is consistent with selection for recessive inactivation of NF1, a typical pattern for a tumor suppressor gene. It also suggests that nongenomedoubled ovarian carcinoma samples evolved through a distinct trajectory, rather than being precursors to doubled samples. If not, many NF1 mutations would be homozygous with multiplicity $>1$ in doubled samples, as is seen for TP53.

Finally, we noted that genome-doubled samples were associated with a significant increase in the age at pathological diagnosis (Fig. 7d) and with a significantly greater incidence of cancer recurrence (Fig. 7e).

\section{DISCUSSION}

Here we report the development of a reliable, high-throughput method to infer absolute homologous copy numbers from tumorderived DNA samples, as well as multiplicity values of point mutations (ABSOLUTE). It may be possible to extend ABSOLUTE to other types of genomic alterations, such as structural rearrangements and small insertions and deletions, although this may require longer sequence reads to ensure accurate sequence alignment.

ABSOLUTE analysis of SCNAs demonstrated that many of the copy-number alterations analyzed were fixed in the cancer lineage represented in the sample (Fig. 3). This was recapitulated in ovarian cancer by somatic point-mutations, many of which were fixed at integer multiplicity (Fig. 4b). Classification of point mutations based on their multiplicities may help distinguish tumor suppressors and oncogenes (Fig. 5d). Knowledge of discrete tumor copy-states, subclonal structure and genome doubling status provides a foundation for further reconstruction of the phylogenetic relationships within a cancer and the temporal sequence by which a given cancer genome arose ${ }^{44-46}$.

ABSOLUTE provides a tool for the design of studies using genomic sequencing to detect variant alleles in cancer tissue samples, based on calculation of sensitivity to detect mutations as a function of sample purity, local copy number and sequencing depth (Supplementary Fig. 7). The high accuracy of tumor purity and ploidy estimates produced by
ABSOLUTE, based on SNP microarray data (Fig. 2), makes it possible to determine the sequencing depth required for a given sample or to select suitable samples given a fixed sequencing depth. Such considerations are vital to the interpretation of subclonal point-mutations (Supplementary Figs. $7 f$ and 10).

Analysis of the predicted absolute allelic copy-number profiles across human cancers produced by ABSOLUTE shed new light on cancer genome evolution. The observed SCNA profiles (Supplementary Fig. 9) were consistent with a common trajectory consisting of an early period of chromosomal instability followed by the emergence of a stable aneuploid clone, as previously described ${ }^{11}$. Our data further indicate that genome doublings occur in a subset of cancer cells already harboring arm-level SCNAs characteristic of the corresponding tumor type. The genomes of these cancers were therefore shaped by selection at chromosomal arm-level resolution before doubling and further clonal outgrowth (Fig. 6d and Supplementary Fig. 10).

These findings are broadly consistent with an earlier interpretation of primary breast cancer FACS/SKY profiles ${ }^{47}$, and has recently been recapitulated in studies of macro-dissected and ploidy-sorted cell populations ${ }^{14}$, and single-cell sequencing ${ }^{15}$ of primary breast tumors. We note that this model represents a departure from the idea that tetraploidization is an initiating event ${ }^{13,26,48-50}$. In addition, the association of genome doubling with epithelial lineage (Fig. 6c) and with age at diagnosis in ovarian carcinoma (Fig. 7d) is consistent with a recently described mechanism linking telomere crisis, DNA damage response, and genome doubling in cultured mouse embryonic fibroblasts ${ }^{49}$.

The analysis of clonality presented in this work offers a path forward for clinical sequencing of cancer, and provides the means to address recently reported concerns regarding intratumor heterogeneity $^{14,15,45,46,51-53}$. Analysis using ABSOLUTE can identify alterations present in all cancer cells contributing to the DNA aliquot (Fig. 1), even if such clonal alterations correspond to the minority of observed mutations. Such alterations are candidate founding oncogenic drivers for a given cancer, which may be the preferred therapeutic targets. Further characterization of subclonal somatic alterations in cancer may become important for understanding variable response to targeted therapeutics, with the clonality of targeted mutations potentially affecting response level.

\section{METHODS}

Methods and any associated references are available in the online version of the paper at http://www.nature.com/naturebiotechnology/.

Note: Supplementary information is available on the Nature Biotechnology website.

\section{ACKNOWLEDGMENTS}

This work was supported by grants from the National Cancer Institute as part of The Cancer Genome Atlas project: U24CA126546 (M.M.), U24CA143867 (M.M.), and U24CA143845 (G.G.). S.L.C. and E.H. were supported by training grant T32 
HG002295 from the National Human Genome Research Institute. In addition, D.P. was supported by the US National Institutes of Health (NIH) - 5R01 GM083299-14; D.A.L. by Department of Defense W81XWH-10-1-0222; T.Z. by NIH/National Institute of General Medical Sciences 5 T32 GM008313; R.B. by NIH K08CA122833 and U54CA143798. B.A.W. was supported by National Research Service Award grant F32CA113126.

\section{AUTHOR CONTRIBUTIONS}

M.M. posed the concept of using allelic copy number analysis to assess tumor genomic purity. G.G. and S.L.C. conceived and designed the analysis. S.L.C. designed and implemented the ABSOLUTE algorithm, and performed data analysis. K.C. assisted with mutation calling and validation. E.H. performed analysis with ASCAT and assisted with multiplicity analysis. A.M. assisted with the determination of statistical power for the ovarian cancer data. T.Z. assisted with analysis of the SCNA length distribution versus genome doubling. R.C.O. and W.W. processed the SNP array experiments. W.W. designed and executed the DNA mixing experiment. H.S. and P.W.L. conceived and executed the leukocyte methylation signature analysis. B.A.W. contributed insight into allelic analysis of SNP array data. D.A.L. managed the FACS analysis of specimens for ploidy determination. All authors contributed to the final manuscript. D.P. and R.B. provided critical review of the manuscript. S.L.C., M.M., G.G. and E.S.L. wrote the manuscript. R.B., M.M. and G.G. provided leadership for the project.

\section{COMPETING FINANCIAL INTERESTS}

The authors declare competing financial interests: details accompany the full-text HTML version of the paper at http://www.nature.com/naturebiotechnology/.

Published online at http://www.nature.com/naturebiotechnology/.

Reprints and permissions information is available online at http://www.nature.com/ reprints/index.html.

1. Pinkel, D. et al. High resolution analysis of DNA copy number variation using comparative genomic hybridization to microarrays. Nat. Genet. 20, 207-211 (1998).

2. Mei, R. et al. Genome-wide detection of allelic imbalance using human SNPs and high-density DNA arrays. Genome Res. 10, 1126-1137 (2000).

3. Lindblad-Toh, K. et al. Loss-of-heterozygosity analysis of small-cell lung carcinomas using single-nucleotide polymorphism arrays. Nat. Biotechnol. 18, 1001-1005 (2000).

4. Zhao, X. et al. An integrated view of copy number and allelic alterations in the cancer genome using single nucleotide polymorphism arrays. Cancer Res. 64, 3060-3071 (2004).

5. Bignell, G.R. et al. High-resolution analysis of DNA copy number using oligonucleotide microarrays. Genome Res. 14, 287-295 (2004).

6. Campbell, P.J. et al. Identification of somatically acquired rearrangements in cancer using genome-wide massively parallel paired-end sequencing. Nat. Genet. 40, 722-729 (2008)

7. Chiang, D.Y. et al. High-resolution mapping of copy-number alterations with massively parallel sequencing. Nat. Methods 6, 99-103 (2009).

8. Kallioniemi, A. et al. Comparative genomic hybridization for molecular cytogenetic analysis of solid tumors. Science 258, 818-821 (1992).

9. Boveri, T. J. Cell Sci. 121 (Suppl.1), 1-84 (2008).

10. Mitelman, F. Recurrent chromosome aberrations in cancer. Mutat. Res. 462, 247-253 (2000)

11. Albertson, D.G., Collins, C., McCormick, F. \& Gray, J.W. Chromosome aberrations in solid tumors. Nat. Genet. 34, 369-376 (2003).

12. Storchova, Z. \& Pellman, D. From polyploidy to aneuploidy, genome instability and cancer. Nat. Rev. Mol. Cell Biol. 5, 45-54 (2004).

13. Storchova, Z. \& Kuffer, C. The consequences of tetraploidy and aneuploidy. J. Cell Sci. 121, 3859-3866 (2008).

14. Navin, N. et al. Inferring tumor progression from genomic heterogeneity. Genome Res. 20, 68-80 (2010).

15. Navin, N. et al. Tumour evolution inferred by single-cell sequencing. Nature $\mathbf{4 7 2}$, 90-94 (2011).

16. Hicks, J. et al. High-resolution ROMA CGH and FISH analysis of aneuploid and diploid breast tumors. Cold Spring Harb. Symp. Quant. Biol. 70, 51-63 (2005).

17. Mullighan, C.G. et al. Genome-wide analysis of genetic alterations in acute lymphoblastic leukaemia. Nature 446, 758-764 (2007).

18. Lyng, H. et al. GeneCount: genome-wide calculation of absolute tumor DNA copy numbers from array comparative genomic hybridization data. Genome Biol. 9, R86 (2008).

19. Hudson, T.J. et al. International network of cancer genome projects. Nature 464, 993-998 (2010).

20. Weir, B.A. et al. Characterizing the cancer genome in lung adenocarcinoma. Nature 450, 893-898 (2007)
21. The Cancer Genome Atlas Research Network. Comprehensive genomic characterization defines human glioblastoma genes and core pathways. Nature 455 1061-1068 (2008).

22. Berger, M.F. et al. The genomic complexity of primary human prostate cancer. Nature 470, 214-220 (2011).

23. Stransky, N. et al. The mutational landscape of head and neck squamous cell carcinoma. Science 333, 1157-1160 (2011).

24. Bass, A.J. et al. Genomic sequencing of colorectal adenocarcinomas identifies a recurrent VTI1A-TCF7L2 fusion. Nat. Genet. 43, 964-968 (2011).

25. Fujiwara, T. et al. Cytokinesis failure generating tetraploids promotes tumorigenesis in p53-null cells. Nature 437, 1043-1047 (2005).

26. Holland, A.J. \& Cleveland, D.W. Boveri revisited: chromosomal instability, aneuploidy and tumorigenesis. Nat. Rev. Mol. Cell Biol. 10, 478-487 (2009).

27. Pleasance, E.D. et al. A comprehensive catalogue of somatic mutations from a human cancer genome. Nature 463, 191-196 (2010).

28. Attiyeh, E.F. et al. Genomic copy number determination in cancer cells from single nucleotide polymorphism microarrays based on quantitative genotyping corrected for aneuploidy. Genome Res. 19, 276-283 (2009).

29. Popova, T. et al. Genome Alteration Print (GAP): a tool to visualize and mine complex cancer genomic profiles obtained by SNP arrays. Genome Biol. 10, R128 (2009).

30. Greenman, C.D. et al. PICNIC: an algorithm to predict absolute allelic copy number variation with microarray cancer data. Biostatistics 11, 164-175 (2010).

31. Van Loo, P. et al. Allele-specific copy number analysis of tumors. Proc. Natl. Acad. Sci. USA 107, 16910-16915 (2010).

32. Yau, C. et al. A statistical approach for detecting genomic aberrations in heterogeneous tumor samples from single nucleotide polymorphism genotyping data. Genome Biol. 11, R92 (2010).

33. Li, A. et al. GPHMM: an integrated hidden Markov model for identification of copy number alteration and loss of heterozygosity in complex tumor samples using whole genome SNP arrays. Nucleic Acids Res. 39, 4928-4941 (2011).

34. The Cancer Genome Atlas Research Network. Integrated genomic analyses of ovarian carcinoma. Nature 474, 609-615 (2011).

35. Roschke, A.V. et al. Karyotypic complexity of the $\mathrm{NCl}-60$ drug-screening panel. Cancer Res. 63, 8634-8647 (2003).

36. Beroukhim, R. et al. The landscape of somatic copy-number alteration across human cancers. Nature 463, 899-905 (2010).

37. Gnirke, A. et al. Solution hybrid selection with ultra-long oligonucleotides for massively parallel targeted sequencing. Nat. Biotechnol. 27, 182-189 (2009).

38. Levanon, K., Crum, C. \& Drapkin, R. New insights into the pathogenesis of serous ovarian cancer and its clinical impact. J. Clin. Oncol. 26, 5284-5293 (2008).

39. Galipeau, P.C. et al. 17p (p53) allelic losses, $4 \mathrm{~N}$ (G2/tetraploid) populations, and progression to aneuploidy in Barrett's esophagus. Proc. Natl. Acad. Sci. USA 93 7081-7084 (1996).

40. Barrett, M.T. et al. Evolution of neoplastic cell lineages in Barrett oesophagus. Nat. Genet. 22, 106-109 (1999).

41. Mermel, C.H. et al. GISTIC2.0 facilitates sensitive and confident localization of the targets of focal somatic copy-number alteration in human cancers. Genome Biol. 12, R41 (2011).

42. Fudenberg, G., Getz, G., Meyerson, M. \& Mirny, L.A. High order chromatin architecture shapes the landscape of chromosomal alterations in cancer. Nat. Biotechnol. 29, 1109-1113 (2011).

43. Ganem, N.J., Godinho, S.A. \& Pellman, D. A mechanism linking extra centrosomes to chromosomal instability. Nature 460, 278-282 (2009).

44. Campbell, P.J. et al. Subclonal phylogenetic structures in cancer revealed by ultradeep sequencing. Proc. Natl. Acad. Sci. USA 105, 13081-13086 (2008).

45. Yachida, S. et al. Distant metastasis occurs late during the genetic evolution of pancreatic cancer. Nature 467, 1114-1117 (2010).

46. Ding, L. et al. Clonal evolution in relapsed acute myeloid leukaemia revealed by whole-genome sequencing. Nature 481, 506-510 (2012).

47. Dutrillaux, B., Gerbault-Seureau, M., Remvikos, Y., Zafrani, B. \& Prieur, M. Breast cancer genetic evolution: I. Data from cytogenetics and DNA content. Breast Cancer Res. Treat. 19, 245-255 (1991).

48. Ganem, N.J., Storchova, Z. \& Pellman, D. Tetraploidy, aneuploidy and cancer. Curr. Opin. Genet. Dev. 17, 157-162 (2007).

49. Davoli, T., Denchi, E.L. \& de Lange, T. Persistent telomere damage induces bypass of mitosis and tetraploidy. Cell 141, 81-93 (2010).

50. Bazeley, P.S. et al. A model for random genetic damage directing selection of diploid or aneuploid tumours. Cell Prolif. 44, 212-223 (2011).

51. Liu, W. et al. Copy number analysis indicates monoclonal origin of lethal metastatic prostate cancer. Nat. Med. 15, 559-565 (2009).

52. Walter, M.J. et al. Clonal architecture of secondary acute myeloid leukemia N. Engl. J. Med. 366, 1090-1098 (2012)

53. Gerlinger, M. et al. Intratumor heterogeneity and branched evolution revealed by multiregion sequencing. N. Engl. J. Med. 366, 883-892 (2012). 


\section{ONLINE METHODS}

Inference of purity, ploidy, and absolute somatic copy-numbers. Homologuespecific copy ratios (HSCRs; copy-ratio estimates of both homologous chromosomes) are preferred for analysis with ABSOLUTE, and were used for all analyses in this study. Although ABSOLUTE can be run on total copy-ratio data (e.g. from array CGH or low-pass sequencing data), we do not present such results here. The use of HSCRs reduces the ambiguity of copy profiles. For example, the total copyratio profile of a sample without SCNAs would be equivalent for ploidy values of $1,2,3$, etc., however the HSCR profile would rule out odd ploidy values, since these would not be consistent with equal homologous copy-numbers. In addition, since subclonal SCNAs will generally affect only one of the two HSCR values in a given genomic segment, the ratio of clonal to subclonal SCNAs genome-wide is generally higher when considering HSCRs rather than only total copy-numbers.

HSCRs were derived from segmental estimates of phased multipoint allelic copy-ratios at heterozygous loci using the program HAPSEG $^{54}$ on data from Affymetrix SNP arrays. As part of this procedure, haplotype panels from population linkage analysis (HAPMAP3) ${ }^{55}$ were used in conjunction with statistical phasing software (BEAGLE) ${ }^{56}$ in order to estimate the phased germline genotypes at SNP markers in each cancer sample. This increased our sensitivity for resolving genotypes, since it naturally exploited the local statistical dependencies between $\mathrm{SNP}^{54}$. In addition, this allowed greater resolution of small differences between homologous copy-ratios, since phase information from allelic imbalance of heterozygous markers due to SCNA could be combined with the statistical phasing from the haplotype panels ${ }^{54}$.

Identification and evaluation of candidate tumor purity and ploidy values. We describe identification of candidate tumor purity and ploidy values and calculation of their SCNA-fit log-likelihood scores using a probabilistic model. This is accomplished by fitting the input HSCR estimates with a Gaussian mixture model, with components centered at the discrete concentrationratios implied by equation (1). The model also supports a moderate fraction of subclonal events which are not restricted to the discrete levels. Candidate solutions are identified by searching for local optima of this likelihood over a large range of purity and ploidy values. This results in a discrete set of candidate solutions with corresponding SCNA-fit likelihoods (equation (1), Fig. 1e-g, Supplementary Fig. 1c-e).

The SCNA-fit scores quantify the evidence for each solution contributed by explanation of the observed HSCRs as integer SCNAs. These computations are independent for each sample. The input data consist of $N$ HSCRs $x_{i}, i \in\{1, \ldots, N\}$. Each of these is observed with standard error $\sigma_{i}$, and corresponds to a genomic fraction denoted $w_{i}$. Each of the $x_{i}$ is assumed to have arisen from either one of $Q$ integer copy-number states: $Q=\{0,1, \ldots, Q-1\}$, or an additional state $Z$ corresponding to subclonal copy-number. We refer to the collection of possible copy-states as $S=Q \cup Z$. We define $Q+1$ indicators $s$ for the copy-state of each segment, with $p\left(s_{i}\right)$ representing the probability of segment $i$ having been generated from state $s \in S$. The integer copy-states of $S$ are indexed by $q \in Q$; the non-integer state is denoted by $z$.

The expected copy-ratio corresponding to each integer copy-number $q(x)$ in a tumor sample is given by equation (1). Note that when homologous copyratios are used, this equation becomes:

$$
\mu_{q}=2\left[\frac{\alpha}{D}\right] q(x)+\left[\frac{2(1-\alpha)}{D}\right]
$$

since HSCRs are measured relative to haploid concentrations, as opposed to the diploid values assumed by equation $1 . D$ is related to tumor purity and ploidy ( $\alpha$ and $\tau$ ) (equation (1), Fig. 1). The observed $x_{i}$ are modeled with a mixture of $Q$ Gaussian components located at $\mu=\left\{\mu_{q \in Q}\right\}$ representing integer copy-states $Q$ and an additional uniform component $Z$. The mixture $Z$ allows segments to be assigned non-integer copy values so that subclonal alterations or artifacts do not dramatically impact the likelihood.

$$
\begin{aligned}
& s_{i} \sim \operatorname{Multinom}\left(p\left(s_{i} \mid w_{i}, \theta\right)\right) \\
& x_{i}= \begin{cases}\mu_{q}+\epsilon_{i} & \text { if } s_{i} \in Q \\
u & \text { if } s_{i}=Z\end{cases} \\
& \epsilon_{i} \sim \mathcal{N}\left(0, \sigma_{i}^{2}+\sigma_{H}^{2}\right) \\
& u \sim \mathcal{U}(d)
\end{aligned}
$$

$\mathcal{N}$ and $\mathcal{U}$ denote the normal and uniform densities, respectively. The free parameter $\sigma_{\mathrm{H}}$ represents sample-level noise in excess of the HSCR standard-error $\sigma_{i}$, which might represent a moderate number of related clones in the malignant cell population, ongoing genomic instability, or excessive noise due to variable experimental conditions. The mixture weights $\theta=\left\{\theta_{s \in S}\right\}$ specify the expected genomic fraction allocated to each copy-state. The parameter $d$ represents the domain of the uniform density, corresponding to the range of plausible copy-ratio values (we used $d=7$ ).

Some complication arises due to the fact that the data consist of copy-ratios calculated from a segmentation of the genome. For consistent interpretation, the mixture weights $P\left(s_{i} \mid w_{i}, \theta\right)$ must be calculated for each segment separately, taking into account the variable genomic fraction $w_{i}$. This is accomplished by constraining the canonical averages of genomic mass allocated to each copystate to match those specified by $\theta$ :

$$
\forall_{s \in S},\left\langle\sum_{i=1}^{N} s_{i} w_{i}\right\rangle_{\mathcal{C}}=\theta_{s}
$$

where $\langle\cdot\rangle_{\mathcal{C}}$ denotes the average over all configurations $\left\{s_{i}\right\}$, weighted by the function $\mathcal{C}=P\left(s_{i} \mid w_{i}, \lambda\right)$. This density corresponds to the maximum entropy distribution over $s$ subject to these constraints:

$$
P\left(s_{i} \mid w_{i}, \lambda\right)=\frac{e^{-\lambda_{s} s^{\#} w_{i}}}{\sum_{k \in S^{e-\lambda_{k} k^{\#}} w_{i}}}
$$

where $s^{\#}$ indicates the order of state $s$ in a sequence of copy-states, beginning with 0 . Values of the $Q$ Lagrange multipliers $\lambda$ are determined via Nelder-Mead optimization of $L_{2}$ loss:

$$
\lambda=\operatorname{argmin}_{\lambda}\left(\sum_{s \in S}\left[\left(\sum_{i=1}^{N}\left[w_{i} P\left(s_{i} \mid w_{i}, \lambda\right)\right]-\theta_{s}\right)^{2}\right]\right)^{\frac{1}{2}}
$$

This approximation allows for robustness of the SCNA-fit score to over-segmentation of the data. The likelihood of a given segment $i$ is then calculated as:

$$
\mathcal{L}\left(x_{i} \mid \mu, \sigma_{i}, \sigma_{H}, \theta, w_{i}\right)=\sum_{q \in Q}\left[P\left(q_{i} \mid w_{i}, \lambda\right) \mathcal{N}\left(x_{i} \mid \mu_{q}, \sigma_{i}^{2}+\sigma_{H}^{2}\right)\right]+P\left(z_{i} \mid w_{i}, \lambda\right) \mathcal{U}(d)
$$

and the full log-likelihood of the data is then:

$$
\sum_{i=1}^{N} \log \mathcal{L}\left(x_{i} \mid \mu, \sigma_{i}, \sigma_{H}, \theta, w_{i}\right)
$$

We define the parameterization

$$
b=2(1-\alpha), \delta_{\tau}=\frac{\alpha}{D}
$$

which determines $\mu$ via equation (3). Candidate purity and ploidy solutions for a tumor sample are identified by optimization of equation (5) with respect to $b$ and $\delta_{\tau}$. Calculation of equation (5) requires estimates of $\theta$ and $\sigma_{H}$, which are not known a priori. We make an approximation (scale-separation) assuming that locations of the modes of equation (5) are invariant to moderate fluctuations in these parameters. A provisional likelihood for each $x_{i}$ may then be calculated by

$$
\mathcal{L}_{P}\left(x_{i} \mid \mu, \sigma_{i}, \sigma\right)=\sum_{q \in Q}\left[\mathcal{N}\left(x_{i} \mid \mu_{q}, \sigma_{i}^{2}+\sigma^{2}\right)\right]+\mathcal{U}(d)
$$

Candidate purity and ploidy solutions are then identified by optimization of

$$
\sum_{i=1}^{N} \log \mathcal{L}_{P}\left(x_{i} \mid \mu, \sigma_{i}, \sigma_{P}\right)
$$

initiated from all points in a regular lattice spanning the domain of $b$ and $\delta_{\tau}$. The parameter $\sigma_{P}$ was set to 0.01 for this study. We verified that the above approximation identified modes equivalent to those obtained through a full Metropolis-Hastings Markov chain Monte Carlo (MCMC) simulation (data not shown). The approximation allows for much simpler computations to be used. 
The SCNA-fit score for each solution is calculated after optimization of $\sigma_{H}$ :

$$
\hat{\sigma}_{H}=\underset{\sigma_{H}}{\operatorname{argmax}} \sum_{i=1}^{N} \log \mathcal{L}\left(x_{i} \mid \hat{\mu}, \sigma_{i}, \sigma_{H}, \theta, w_{i}\right)
$$

with the elements of $\theta$ calculated for each value of $\sigma_{\mathrm{H}}$ by:

$$
\hat{\theta}_{q}=\sum_{i=1}^{N} w_{i} \frac{\mathcal{N}\left(x_{i} \mid \hat{\mu}_{q}, \sigma_{i}^{2}+\sigma_{H}^{2}\right)}{\mathcal{L}_{P}\left(x_{i} \mid \hat{\mu}, \sigma_{i}, \sigma_{H}\right)} ; \hat{\theta}_{z}=\sum_{i=1}^{N} w_{i} \frac{\mathcal{U}(d)}{\mathcal{L}_{P}\left(x_{i} \mid \hat{\mu}, \sigma_{i}, \sigma_{H}\right)}
$$

The final calculation of the SCNA-fit log-likelihood for each mode is obtained by inserting $\hat{\boldsymbol{\mu}}, \hat{\boldsymbol{\theta}}$ and $\hat{\sigma}_{\mathrm{H}}$ into equation (5). Estimates of the copy-state indicators for each segment are calculated as:

$$
\begin{aligned}
& \hat{\boldsymbol{q}}_{i}=P\left(q_{i} \mid w_{i}, \lambda\right) \frac{\mathcal{N}\left(x_{i} \mid \hat{\mu}_{q}, \sigma_{i}^{2}+\hat{\sigma}_{H}^{2}\right)}{\mathcal{L}\left(x_{i} \mid \hat{\mu}, \sigma_{i}, \hat{\sigma}_{H}, \hat{\theta}, w_{i}\right)} \\
& \hat{z}_{i}=P\left(z_{i} \mid w_{i}, \lambda\right) \frac{\mathcal{U}(d)}{\mathcal{L}\left(x_{i} \mid \hat{\mu}, \sigma_{i}, \hat{\sigma}_{H}, \hat{\theta}, w_{i}\right)}
\end{aligned}
$$

Note that each $\hat{\boldsymbol{q}}_{i}$ is a vector representing the posterior probability of each $Q \in Q$ integer copy-states, corresponding to the copy-ratios (locations) $\mu$.

Genome-wide absolute copy-profiles are over-determined with respect to DNA ploidy estimates. An alternate estimate of ploidy may be calculated as the expected absolute copy-number over the genome:

$$
\hat{\tau}_{g}=\sum_{i=1}^{N}\left(w_{i} \sum_{q=0}^{Q-1} q \hat{\boldsymbol{q}}_{i q}\right)
$$

By definition, this quantity $\left(\hat{\tau}_{g}\right)$ is an alternate estimate of cancer ploidy (note an additional factor of 2 is added when HSCRs are used). Because $\hat{\tau}_{g}$ is a weighted average over discrete states in the modeled data, it is expected to be more robust to experimental fluctuations that shift or scale the copy-profile slightly. Note that, for this computation, the $\hat{q}_{i j}$ were calculated with $\hat{\theta}_{z}=0$, so that the above expectation is over integer states only.

We verified that these estimates were generally close to the values of $\hat{\tau}$ obtained by optimization of the SCNA-fit likelihood (RMSE $=0.26$, Supplementary Fig. 11a). However, we noted a relationship between the level of discordance between ploidy estimators and the mean of the calibrated data (Supplementary Fig. 11b). Noting that correctly calibrated copy-ratio data always has mean $=1$, we examined whether the miscalibration was due to scaling bias in the data. We found that this model explained nearly two thirds of the discordance between the two estimates (corrected RMSE $=0.09$, Supplementary Fig. 11c), by which we inferred that scaling biases dominated our miscalibration. This is significant, as such biases do not effect the estimation of tumor purity (Supplementary Fig. 11).

Two additional transformations of the copy-state locations $\mu$ are used when copy-ratios are measured using microarrays. The first of these accounts for the effect of attenuation with an isothermal adsorbtion model?:

$$
g(x)=\frac{x(1+\hat{\phi})}{(1+x \hat{\phi})}
$$

where the value $\hat{\phi}$ parameterizes the attenuation response in a given sample, and is estimated via HAPSEG. The second transformation is a variance stabilization for microarray data adapted from ${ }^{57}$ :

$$
h(x)=\operatorname{arcsinh}\left(\frac{x e^{\sigma_{\eta}^{2}}-1}{\sigma_{\epsilon}}\right)
$$

where $\sigma_{\eta}$ and $\sigma_{\epsilon}$ represent multiplicative and additive noise scales for each microarray, estimated by HAPSEG. This transformation is applied to the marker-level data during estimation of the $x_{i}$ values, after which their distribution is approximately normal. The normal mixture component specified in
(4) then becomes $h\left(x_{i}\right)=h\left(g\left(\mu_{q}\right)\right)+\epsilon_{i}$, and the corresponding likelihood calculations are performed under these transformations.

Karyotype models. Additional information is generally required in order to reliably select the correct tumor purity and ploidy solution from the set of candidates identified by fitting the model in (4). In a given tumor sample, several combinations of theoretically possible purity, ploidy, and copy number values may map to equivalent copy ratios (Fig. 1e-g, Supplementary Fig. 1). Furthermore, the presence of subclonal SCNAs may result in a spuriously high ploidy solution with an implausible karyotype receiving a greater SCNA-fit likelihood by over-discretizing the copy profile, allowing their assignment to integer copy-levels (Supplementary Fig. 1c-e).

ABSOLUTE models common cancer karyotypes by grouping tumor sets according to similarities in their absolute homologous copy-number profiles (Supplementary Fig. 2). These models are constructed directly from the tumor data in a 'boot-strapping' fashion, whereby a subset of tumors with relatively unambiguous profiles (e.g., due to high purity values) is used initialize the models, iteratively allowing more tumors to be called, etc. Previous cytogenetic characterizations of human cancer were used to guide this process ${ }^{13}$. These models enable calculation of a karyotype likelihood, for each candidate purity/ ploidy solution, reflecting the similarity of the corresponding karyotype to models associated with the specified disease of the input tumor sample (8). Integration of the SCNA-fit and karyotype likelihoods favors robust and unambiguous identification of the correct purity and ploidy values in many tumor samples ( Fig. 1g, Supplementary Fig. 1e).The selection of a solution implying a less common karyotype requires greater evidence from the SCNA-fit of the copy profile.

Prior knowledge of karyotypes characteristic of a particular disease is summarized as a mixture of $K$ multivariate multinomial distributions over the integer homologous copy-states $Q=[0,7]$ of each chromosome arm. For a given candidate purity and ploidy solution, the corresponding segmental copy-state indicators for each segment $i, \hat{q}_{i j}$, are summarized into estimates of the $J$ arm-level homologous copy-numbers, denoted $\hat{C}$. The karyotype loglikelihood score is calculated as:

$$
\mathcal{L}_{K}(\hat{\boldsymbol{C}} \mid \boldsymbol{K})=\log \sum_{i=1}^{K}\left[w_{i} \prod_{j=1}^{J} \prod_{q \in \mathbf{Q}} \boldsymbol{K}_{i j q}^{\hat{\boldsymbol{C}}_{j q}}\right]
$$

where $w_{i}$ denotes the weight of each mixture component. The karyotype models $\boldsymbol{K}_{i}$ are $J \times Q$ SCNA probability matrices obtained by clustering arm-level homologous copy-states of modeled copy-profiles using the standard expectation-maximization (EM) algorithm ${ }^{58}$ for multinomial mixtures. This calculation identifies groups of disease subtypes with similar genomic copy profiles (Supplementary Fig. 2). Note that copy-states for both homologues of each arm are modeled $(J=78)$. Karyotype scores for samples with only total copyratio data are calculated using convolution of the multinomial probabilities for the two homologous chromosomes.

The number of clusters $K$ for each disease was chosen by minimizing the Bayesian information criterion (BIC) complexity penalty: $-2 \hat{L}_{k}+K J \log (N)$, where $\hat{L}_{k}$ indicates the sum of $\mathcal{L}_{K}$ values over the $N$ input samples, computed using $K$ clusters. In order to avoid local minima, the EM algorithm was run 25 times for each value of $K \in[2,8]$ with randomized starting points and the best model was retained.

The models were constructed in a semi-automated fashion by seeding with relatively unambiguous copy-profiles. As tumors were added, the use of recurrent karyotypes clearly identified the correct solutions of additional samples, etc. For example, $\mathrm{LOH}$ of chr17 occurs in nearly $100 \%$ of ovarian carcinoma samples ${ }^{34}$, allowing the model to learn that solutions implying $\mathrm{LOH}$ of chr17 are likely to be correct. In total, models for 14 disease types were created. Diseases with fewer than 40 samples called by ABSOLUTE were omitted 
from this procedure. In addition, a "master" model was created by combining called primary cancer profiles. This model was used for diseases with no specific karyotype model.

Limitations of joint purity/ploidy inference from copy-profile data. Accurate calibration of both the SCNA-fit and karyotype models to the true level of certainty implied by the data would allow for assignment of probabilities to each candidate solution; we do not believe that the models we have presented here sufficiently capture the complexity of cancer genomes to allow for such interpretations. Even with manual review, analysis with ABSOLUTE may occasionally result in incorrect interpretations, for example genome-doubling without subsequent detectable gains or losses would result in a solution implying half the true ploidy value, which in some cases may correspond to a plausible karyotype model. Alternately, when multiple subclonal SCNAs appear close to the midpoints between adjacent clonal peaks, a solution implying twice the true ploidy may be chosen. We note that samples with no reliably detected SCNAs could not be called in our framework (ploidy $2 n$ or $4 n$; purity undetermined). Such samples were therefore excluded from downstream analysis (see below). Estimation of inference error-rate requires independent measurement of sample ploidy. Further validation experiments in diverse tumor types will help to clarify any disease specific caveats.

We note that the use of somatic mutation allelic-fractions, combined with the SCNA copy-ratios, generally allows for increased sensitivity for samples with few SCNAs. In addition, the mutation data helps distinguish genomedoubling ambiguity in purity/ploidy estimation, although it does not inform ambiguities of the type $b^{\prime}=b+2(1-\alpha) / D$ (Fig. 1f, Supplementary Fig. 1d, equation (1)). Thus, combined analysis generally facilitates obtaining higher call-rates using ABSOLUTE (not shown).

Fortunately, many samples in our pan-cancer SNP array dataset were consistent with frequent SCNA both before and after genome doubling, enabling unambiguous inference for many samples without use of somatic pointmutation data. This aspect of cancer genome evolution was noted previously in breast cancer cytogenetic data ${ }^{47}$. We note that manual review of ABSOLUTE results was performed prior to generation of the FACS validation data or analysis of the NCI60 cell-line ploidy estimates (Fig. 2a,b).

Identification of samples refractory to purity/ploidy inference. In order to facilitate rapid analysis of many cancer samples used in this study, ABSOLUTE was programed to automatically identify copy profiles that cannot be reliably called and to classify them into informative failure categories (Fig. 3a), which were defined by the following criteria. Define $\hat{\boldsymbol{m}}$ as the sorted vector of posterior genome-wide copy-state allocations $(\hat{\theta})$, so that $\hat{m}_{1}$ represents the greatest element of $\hat{\boldsymbol{\theta}}$ (the modal copy-state). This vector was constructed with $\theta_{0}$ replaced by 0 if $\theta_{0}<0.01$ and $b<0.15$, so that germline copy-number variants (CNVs) or regions of inherited homozygosity are not confused with small SCNAs implying very pure samples. The categories are then:

1. non-aberrant: $\hat{m}_{3}<0.001, \hat{m}_{2}<0.005, \hat{\sigma}_{H}<0.02$

2. insufficient purity: $\hat{m}_{3}<0.001, \hat{m}_{2}<0.005, \hat{\sigma}_{H} \geq 0.02$

3. polygenomic: $\hat{\theta}_{z}>0.2$.

These criteria were applied to the top-ranked mode for each sample (combined SCNA-fit and karyotype scores). Several examples of each outcome are shown in Supplementary Figure 5. The above designations led to reasonably good concordance of automated calls with those obtained after manual review. We note that the use of somatic point-mutation data increases the calling sensitivity within these sample categories.

Cancer cell-line DNA mixing experiment. DNA extracted from two cancer cell lines (HCC38, HCC1143) was mixed with DNA from matched
B-lymphocyte cell lines (HCC38BL, HCC1143BL) in various proportions, and hybridized to Affymetrix $250 \mathrm{~K}$ Sty SNP arrays. Stock DNA aliquots were created for each cell-line by normalization of DNA concentration to $50 \mathrm{ng} / \mu \mathrm{l}$. Mixing of cancer and matched B-lymphocyte DNA to each required mixing fraction was done by volume.

FACS analysis of primary tumor samples. Formalin-fixed and paraffinembedded blocks from ovarian serous carcinoma cases were available from tumor-sections corresponding to the frozen blocks from which DNA-aliquots were obtained for SNP-array hybridization. Multiple curls containing at least $70 \%$ tumor cell nuclei were cut to an aggregate thickness of $150 \mu \mathrm{m}$. Sections were disaggregated and labeled with propidium iodide (DNA stain). FACS was performed to determine ploidy.

Determination of tumor purity via pathology review. Frozen ovarian serous cystadenocarcinoma specimens were collected from multiple hospital tissue banks and maintained frozen in liquid nitrogen vapors. A tissue portion was created with two flanking H\&E slides (arbitrarily named top and bottom) as follows: tissues were mounted in optimal cutting temperature media (OCT) and brought to $-20{ }^{\circ} \mathrm{C}$. A $4 \mu \mathrm{m}$ frozen section (top slide) was cut with a cryostat (Leica Microsystems, Wetzlar, Germany). A specimen for molecular extraction was created by shaving $100 \mathrm{mg}$ of tumor tissue from the tissue face with a scalpel, then a second $4 \mu \mathrm{m}$ frozen section was cut (bottom slide). An H\&E stain was conducted on both slide tissue sections using an Autostainer $\mathrm{XL}$ with integrated coverslipper (Leica). Digital images of slides were created at $20 \times$ resolution using a Scanscope XT (Aperio, Vista, CA, USA). Boardcertified pathologists conducted the pathology review remotely via ImageScope software (Aperio). Pathologists initially reviewed each slide at low magnification to determine low power microscopic morphology, then increased magnification to $20 \times$ and reviewed 10 representative high power fields on each slide. Diagnosis of ovarian serous cystadenocarcinoma was verified, and tumor purity was determined as the proportion of tumor nuclei present compared to the total nuclei present on the slide. The tumor purity of the extracted specimen was calculated as the average purity score of the top and bottom slides. Quality control included a random review of $10 \%$ of slides by a second pathologist to verify consistency of reads.

Leukocyte methylation signature. DNA methylation data for 489 high stage, high grade serous ovarian tumors and eight normal fallopian tube samples was obtained from http://tcga.cancer.gov/dataportal/. In addition, buffy coat samples from two female individuals were obtained. All data were generated with Illumina Infinium HumanMethylation27 arrays, which interrogate 27,578 CpG sites located in proximity to the transcription start sites of 14,475 consensus coding sequencing in the NCBI Database (Genome Build 36). The level of DNA methylation at each probe was summarized with beta values ranging from 0 (unmethylated) to 1 (methylated) ${ }^{59}$.

The leukocyte methylation signature was derived as follows. Each probe was ranked by the difference in mean beta value in buffy coat and fallopian tube samples. We retained the 100 probes with the largest positive difference and the 100 with the largest negative difference between mean DNA methylation in normal fallopian tube tissues and peripheral blood leukocytes, designated $B C$ and $F T$ (buffy coat and fallopian tube enriched, respectively). Let $T_{i k}$ denote the beta value for probe $k$ in tumor sample $i$. Let $B_{k}$ denote the average beta value of buffy coat samples for each probe. Let $T_{k}$ denote the minimum observed beta value across all tumor samples for the $B C$ probes and the maximum for the FT probes. Denote by $f_{B}$ the fraction of buffy coat components in the sample, then we have the following equation for each probe: $T_{i k}=B_{k} f_{B}+T_{k}\left(1-f_{B}\right)$. Solving this equation for $f_{B}$ gives: $f_{B}=\left(T_{i k}-T_{k}\right) /\left(B_{k}-T_{k}\right)$. The values of $f_{B}$ for each of the 200 probes in the signature were calculated and a kernel density estimate was obtained. The leukocyte signature was then calculated as the mode of this density estimate. 
Selection of data sets. We analyzed 2,445 Affymetrix 250 K Sty SNP samples from a previous pan cancer survey ${ }^{36}$ containing 3,131 cancer samples. Because our processing of the data required use of the Birdseed algorithm ${ }^{60}$, external data sets lacking diploid PCR controls could not be used. In addition, cancer types with fewer than 20 samples were excluded. In addition, 680 Affymetrix SNP6.0 samples were taken from the TCGA GBM ${ }^{21}$ and $\mathrm{HGS}-\mathrm{OvCa}^{34}$ studies, as well as 30 cell-line samples, bringing total sample count to 3,155 . The complete table of cancer samples analyzed is available as Supplementary Table 1. The complete table of ABSOLUTE results is available as Supplementary Tables $\mathbf{1}$ and $\mathbf{2}$.

Power calculation for somatic mutation detection in cancer tissue samples. We develop a framework for calculation of statistical power for the detection of mutations. Power to detect a variant depends on the allelic fraction $f$ and local depth of coverage $n$. To calculate power, we model the idealized scenario in which random sequencing errors occur uniformly with rate $\in$. We calculate a minimum number of supporting reads $k$ such that the probability of observing $k$ or more identical non-reference reads due to sequencing error is less than a defined false-positive rate (FPR):

$$
k=\underset{m}{\arg \min } \mid P(m) \leq \mathrm{FPR}
$$

where

$$
P(m)= \begin{cases}1 & \text { if } m=0 \\ 1-\sum_{i=0}^{m-1} \operatorname{Binom}(i \mid n, \in / 3) & \text { if } m \geq 1\end{cases}
$$

Variants with $\geq k$ supporting reads are then considered detected. We specified the sequencing error rate $\in=1 \times 10^{-3}$ and FPR $=5 \times 10^{-7}$ for all computations in this study. Power is then calculated as:

$$
\operatorname{Pow}(n, f)=1-\sum_{i=0}^{k-1} \operatorname{Binom}(i \mid n, f)+d \operatorname{Binom}(k \mid n, f)
$$

where

$$
d=\frac{F P R-P(k)}{P(k-1)-P(k)}
$$

We consider the case of detecting clonal somatic variants present at a single copy per cancer cell in cancer-tissue derived DNA samples. Given estimates of purity $(\alpha)$ and local absolute copy-number $\left(q_{t}\right)$, the allelic fraction of such variants is:

$$
\delta=\frac{\alpha}{2(1-\alpha)+\alpha q_{t}}
$$

Power is calculated in such cases as $\operatorname{Pow}(n, \delta)$.

In order to simplify the relationship between power and tumor purity/ ploidy for presentation in Supplementary Figure 7, we considered the detection power of the expected locus, over the genome-wide copy average. Power as such is determined by the sample allelic index $\delta_{\tau}=\alpha / D$, which is solely a function of tumor purity/ploidy (equation 1). Expected power is obtained by using allelic fraction $f=\delta_{\tau}$ in equation (9). This calculation differs only in the substitution of expected genomic copy-number, i.e. ploidy $(\tau)$, for the local copy-number $q_{t}$ in equation (10).

Power for expected subclonal variants present in fraction $s_{f}$ of cancer cells is given by $\operatorname{Pow}\left(n, s_{f} \delta_{\tau}\right)$. This calculation was used for Supplementary Figure 7 c,e. Local copy calculations using $\operatorname{Pow}\left(n, s_{f} \delta\right)$ were used for Supplementary Figures $7 \mathrm{f}$ and $\mathbf{1 2}$.

Detection of somatic point mutations in ovarian carcinoma. We analyzed whole-exome hybrid capture Illumina sequencing (WES) ${ }^{37}$ data from 214 ovarian carcinoma tumor-normal pairs previously analyzed by the TCGA consortium ${ }^{34}$. We used the program muTect (K. Cibulskis et al., in preparation) We have used a newer version of the program muTect than used in previous analysis of this data ${ }^{34}$. The primary improvement in the new version is a reduction in the prior that somatic mutations be at an allelic fraction of 0.5 , allowing greater sensitivity at low allelic-fraction mutations, such as clonal events in impure samples, or to subclonal mutations. This procedure resulted in 29,268 somatic mutations.

Inference of point mutation multiplicity. We develop a probabilistic model for inference of the integer multiplicities for both germline and somatic variants, based on knowledge of tumor purity and genome-wide absolute copy-numbers. Denote the absolute homologous copy-numbers at a mutant locus as $\mathrm{q}_{1}$ and $q_{2}$, with $q_{1} \leq q_{2}$. The possible multiplicities of germline variants are then:

$$
\mathbf{g}_{\mathbf{q}}=\left\{q_{1}, q_{2}, q_{t}\right\}
$$

where $q_{t}=q_{1}+q_{2}$. Under the assumption that all somatic point-mutations arise uniquely on a single haplotype, the possible multiplicities are:

$$
\mathbf{s}_{\mathbf{q}}=\left\{1, \ldots, q_{2}\right\}
$$

Note that when only total copy-ratio data are available, $q_{2}$ above is unknown, and $q_{t}$ is used instead.

Germline mutations are generally present in both the cancer and normal cell populations, with somatic copy-number alterations affecting the allelic fraction. A heterozygous variant in the germline, with multiplicity $g_{q}$ in the cancer genome, has allelic fraction:

$$
f_{g_{q}}=\frac{(1-\alpha)+\alpha g_{q}}{2(1-\alpha)+\alpha q_{t}}
$$

whereas the allelic fraction of homozygous germline variants is 1 regardless of $\alpha$. For somatic point mutations, the expected allelic fraction at multiplicity $s_{q}$ is $f_{s q}=s_{q} \delta$, with $\delta$ as in equation (10).

Consider an observed somatic point-mutation of unknown copy $s_{q} \in \mathbf{s}_{\mathbf{q}}$, observed allelic fraction $\hat{f}$, and with $n$ total reads covering the locus. The complete likelihood of $\hat{f}$ may be represented as a mixture of Beta distributions corresponding to each element of $\mathbf{s}_{\mathbf{q}}$, plus an additional component $S$ corresponding to subclonal states:

$$
\begin{aligned}
\mathcal{L}_{m}\left(\hat{f} \mid n, \mathbf{s}_{\mathbf{q}}, \mathbf{w}_{\mathbf{q}},\right. & \left.w_{s_{c}}\right) \\
= & \sum_{s_{q} \in \mathbf{s}_{\mathbf{q}}}\left[w_{s_{q}} \operatorname{Beta}\left(f_{s_{q}} \mid n \hat{f}+1, n(1-\hat{f})+1\right)\right] \\
& +w_{s_{c}} S(\hat{f} \mid n, \lambda)
\end{aligned}
$$

where $w_{s_{q}} \in \mathbf{w}_{\mathbf{q}}$ specify mixture weights for each state in $s_{q}$ and $w_{s_{c}}$ specifies the subclonal component weight. The subclonal component $S$ is specified by composing a Beta distribution (modeling sampling noise) with an exponential distribution over subclonal cancer-cell fractions, having a single parameter $\lambda$ :

$$
S(\hat{f} \mid n, \lambda)=\int_{0}^{1} \operatorname{Beta}(f \mid n \hat{f}+1, n(1-\hat{f})+1) \operatorname{Exp}(f / \delta \mid \lambda) \delta^{-1} d f
$$

Note the change of coordinates in the exponential component using $\delta$; this allows modeling in consistent units of cancer-cell fractions, regardless of tumor purity and local copy-number (note this distribution is renormalized on the unit interval). The probability of a given integer copy-state $s_{q}$ may then be calculated as:

$$
\hat{s}_{q}=\frac{w_{s_{q}}}{q_{2}} \frac{\operatorname{Beta}\left(f_{s_{q}} \mid n \hat{f}+1, n(1-\hat{f})+1\right)}{\mathcal{L}_{m}\left(\hat{f} \mid n, \mathbf{s}_{\mathbf{q}}, \mathbf{w}_{\mathbf{q}}, w_{s_{c}}\right)}
$$

Similarly, the probability that a given mutation is subclonal is calculated as:

$$
\hat{s}_{c}=w_{s_{c}} q_{2} \frac{S(\hat{f} \mid n, \lambda)}{\mathcal{L}_{m}\left(\hat{f} \mid n, \mathbf{s}_{\mathbf{q}}, \mathbf{w}_{\mathbf{q}}, w_{s_{c}}\right)}
$$


For the computations in this study, we fixed $\lambda=25, w_{s_{q}}$ to 0.25 , and $w_{s_{\mathcal{C}}}$ to 0.75 , which produced a fit to the combined-sample mutation-fraction distribution (Fig. 4b). The results presented in Figure 4 were robust to various settings.

Optimization of mixture components weights corresponding to integer somatic multiplicities may be accomplished in a manner similar to that described for the SNCA mixture model in equation (6). A Dirichlet prior may be specified as a vector of pseudo-counts equivalent to prior observations of each multiplicity value. Weights are then calculated as the mode of the posterior Dirichlet calculated from the observed counts. These computations are used to calculate a mutation-score likelihood for each purity ploidy mode when ABSOLUTE is run with paired SCNA and somatic point mutation data.

Simulation of cancer-genome evolution to support genome-doubling inferences. A simple simulation was performed to obtain $P$-values for the probability that an observed configuration of homologous copy-numbers could be produced from a serial process of independent gains and losses. Genome-wide homologous copy-numbers are summarized at chromosome-arm resolution as integer gains/losses (total of 78 states). We then fix the total number of gains/ losses $\mathrm{N}$ for the sample, and calculate rates for each arm, which are normalized to probabilities. Simulation of the sample is performed by independently sampling $N$ gains and losses from these probabilities. This was repeated 1,000 times for each sample, keeping track of the number of times $M$ that the extent of even high homologous copy-number present in the observed sample was attained or exceeded. The $P$-value is then: $P=\frac{M}{1,000}$, if $M>0$, otherwise $P<0.0001$.

54. Carter, S.L, Meyerson, M., \& Getz, G. Accurate estimation of homologue-specific DNA concentration ratios in cancer samples allows long-range haplotyping. Preprint at http://precedings.nature.com/documents/6494/version/1/ (2011).

55. Altshuler, D.M. et al. Integrating common and rare genetic variation in diverse human populations. Nature 467, 52-58 (2010).

56. Browning, B.L. \& Yu, Z. Simultaneous genotype calling and haplotype phasing improves genotype accuracy and reduces false-positive associations for genome-wide association studies. Am. J. Hum. Genet. 85, 847-861 (2009).

57. Huber, W., von Heydebreck, A., Sultmann, H., Poustka, A. \& Vingron, M. Variance stabilization applied to microarray data calibration and to the quantification of differential expression. Bioinformatics 18 (suppl 1), S96-S104 (2002).

58. Dempster, A.P., Laird, N.M. \& Rubin, D.B. Maximum Likelihood from Incomplete Data via the EM Algorithm. J. Roy. Stat. Soc. Ser. B 39, 1-38 (1977).

59. Noushmehr, H. et al. Identification of a CpG island methylator phenotype that defines a distinct subgroup of glioma. Cancer Cell 17, 510-522 (2010).

60. Korn, J.M. et al. Integrated genotype calling and association analysis of SNPs, common copy number polymorphisms and rare CNVs. Nat. Genet. 40, 1253-1260 (2008). 